COMMUNICATIONS IN

ANALYSIS AND GEOMETRY

Volume 13, Number 3, 479-510, 2005

\title{
The Continuity of Convex Cores With Respect to the Geometric Topology
}

\author{
KEN'ICHI OHSHIKA \\ Dedicated to Professor Yukio Matsumoto \\ on the occasion of his sixtieth birthday
}

\section{Introduction.}

The volume of the convex core, the bottom of the spectrum of the Laplacian, and the Hausdorff dimension of the limit set are important invariants of hyperbolic 3-manifolds/Kleinian groups, which are closely related to one another. The behaviour of such invariants with respect to the convergence of Kleinian groups is an interesting problem to study, and also, it is expected that these invariants would be useful to investigate the topological structure of deformation spaces. The behaviour of these invariants with respect to algebraic and geometric convergence of Kleinian groups has been studied by Canary, Taylor, McMullen, among others. (See [10], [27], [31].)

For studying this problem, it is essential to see whether convex cores of the corresponding hyperbolic 3-manifolds converge to the convex core of the limit geometrically in the sense of Gromov. Consider a sequence of Kleinian groups $\left\{G_{i}\right\}$ converging geometrically to $G_{\infty}$. The limit set $\Lambda_{G}$ of a Kleinian group $G$ coincides with the intersection of $S_{\infty}^{2}$ and the closure of the Nielsen convex hull of $G$ in $\mathbf{H}^{3} \cup S_{\infty}^{2}$. If the convex cores $C\left(\mathbf{H}^{3} / G_{i}\right)$ converge geometrically to $C\left(\mathbf{H}^{3} / G_{\infty}\right)$, then the Nielsen convex hulls of $G_{i}$ converge to that of $G_{\infty}$ with respect to the Hausdorff topology of $\mathbf{H}^{3}$. This implies that the limit sets $\Lambda_{G_{i}}$ converge to $\Lambda_{G_{\infty}}$ with respect to the Hausdorff topology on $S_{\infty}^{2}$. Conversely, as was shown by Bowditch [4], if the limit sets $\Lambda_{G_{i}}$ converge to $\Lambda_{G_{\infty}}$, then the convex cores $C\left(\mathbf{H}^{3} / G_{i}\right)$ converge to $C\left(\mathbf{H}^{3} / G_{\infty}\right)$ geometrically in the sense of Gromov. We are interested in the following question. Do the convex cores of $\mathbf{H}^{3} / G_{i}$ converge geometrically to that of $\mathbf{H}^{3} / G_{\infty}$ ? Or equivalently, do the limit sets $\Lambda_{G_{i}}$ converge to $\Lambda_{G_{\infty}}$ with respect to the Hausdorff topology? 
The most interesting case for this problem is when $\left\{G_{i}\right\}$ is a sequence of Kleinian groups with an isomorphism from a fixed Kleinian group $\phi_{i}: G \rightarrow G_{i}$ converging also algebraically to an isomorphism $\psi: G \rightarrow \Gamma$. Jørgensen and Marden in [21] showed that in this situation, if the geometric limit $G_{\infty}$ is geometrically finite, then $\left\{\Lambda_{G_{i}}\right\}$ converges to $\Lambda_{G_{\infty}}$ with respect to the Hausdorff topology. On the other hand, Anderson and Canary proved, in [1], that in this setting, if $\psi$ is type-preserving, i.e. if both $\psi$ and $\psi^{-1}$ preserve parabolicity, and $\Lambda_{\Gamma} \neq S_{\infty}^{2}$, then $\Gamma$ coincides with $G_{\infty}$ and $\left\{\Lambda_{G_{i}}\right\}$ converges to $\Lambda_{G_{\infty}}=\Lambda_{\Gamma}$ with respect to the Hausdorff topology. Since it is easy to see that if $\Lambda_{\Gamma}=S_{\infty}^{2}$, then $\left\{\Lambda_{G_{i}}\right\}$ converges to $\Lambda_{G_{\infty}}$, which must be equal to $\Lambda_{\Gamma}$, this work answers the question posed above affirmatively for the case when the algebraic limit $\Gamma$ has no additional parabolic elements. (Evans' theorem in [14] makes it possible to generalize this result to the case when the isomorphism from $G$ to $\Gamma$ is weakly type-preserving.)

In this paper, we shall study the same problem without assumption that the geometric limit is geometrically finite, nor the assumption that the algebraic limit has no additional parabolic elements. We shall prove that even, then the convex cores converge to that of geometric limit geometrically if the group is assumed to be freely indecomposable relative to the parabolic subgroups and without double trouble. Here, we say that a Kleinian group $G$ is freely indecomposable relative to the parabolic subgroups when for any non-trivial free product decomposition of $G$ into $A * B$, there is a parabolic element none of whose conjugates are contained in the factors $A, B$. We say that $G$ has double trouble when there are two simple closed curves on the boundary of a compact core $C$ of $\mathbf{H}^{3} / G$ which are not homotopic to each other on $\partial C$, but both represent the same parabolic class contained in a parabolic group isomorphic to $\mathbf{Z} \times \mathbf{Z}$.

Theorem 1.1. Let $G$ be a geometrically finite torsion-free Kleinian group. Suppose that $G$ is freely indecomposable relative to the parabolic groups and does not have double trouble. Let $\left\{\left(G_{i}, \phi_{i}\right)\right\}$ be a sequence of (possibly geometrically infinite) Kleinian groups with isomorphisms $\phi_{i}: G \rightarrow G_{i}$ mapping parabolic elements to parabolic elements, which converge algebraically to a Kleinian group $(\Gamma, \psi)$. Take conjugates of $G_{i}$ so that $\left\{\phi_{i}\right\}$ converges to $\psi$ as representations and let $G_{\infty}$ be a geometric limit of $\left\{G_{i}\right\}$, which is known to exist if we extract a subsequence. Then, the convex cores $C\left(\mathbf{H}^{3} / G_{i}\right)$ converge geometrically to the convex core $C\left(\mathbf{H}^{3} / G_{\infty}\right)$ in the sense of Gromov as subspaces of $\mathbf{H}^{3} / G_{i}$ and $\mathbf{H}^{3} / G_{\infty}$ respectively. 
It should be noted that Fan's work in [15], combined with the results of Kerckhoff-Thurston's [23] and Bowditch [3], implies a result similar to the theorem above in the case when $\mathbf{H}^{3} / G$ is homeomorphic to the interior of either an $I$-bundle over a closed surface or an acylindrical 3-manifold. In this line of argument, we do not need to assume that $G_{i}$ converges algebraically.

As was remarked before, the geometric convergence of the convex cores implies the Hausdorff convergence of the limit sets.

Corollary 1.2. In the situation of Theorem 1.1, the limit sets $\Lambda_{G_{i}}$ converge to $\Lambda_{G_{\infty}}$ with respect to the Hausdorff topology on $S_{\infty}^{2}$.

Let us sketch an outline of the proof of the theorem. Since $\left\{G_{i}\right\}$ converges to $G_{\infty}$ geometrically, by taking a point $x \in \mathbf{H}^{3}$ and taking as basepoints its projections $x_{i} \in \mathbf{H}^{3} / G_{i}$ and $x_{\infty} \in \mathbf{H}^{3} / G_{\infty}$ by the universal covering maps, we have geometric convergence $\left(\mathbf{H}^{3} / G_{i}, x_{i}\right) \rightarrow\left(\mathbf{H}^{3} / G_{\infty}, x_{\infty}\right)$ in the sense of Gromov. Therefore, we have an approximate isometry between metric balls with radii $r_{i}, r_{i}^{\prime}$, which we denote by $\rho_{i}: B_{r_{i}}\left(\mathbf{H}^{3} / G_{i}, x_{i}\right) \rightarrow B_{r_{i}^{\prime}}\left(\mathbf{H}^{3} / G_{\infty}, x_{\infty}\right)$ with $r_{i}, r_{i}^{\prime} \rightarrow \infty$. We can easily see that $\left\{\left(C\left(\mathbf{H}^{3} / G_{i}\right), x_{i}\right)\right\}$ converges geometrically to a submanifold $C_{\infty}$ of $\mathbf{H}^{3} / G_{\infty}$ containing $C\left(\mathbf{H}^{3} / G_{\infty}\right)$. What we are going to prove is the inclusion of the opposite direction.

The proof is by contradiction. If $C_{\infty}$ has a point not contained in the convex core $C\left(\mathbf{H}^{3} / G_{\infty}\right)$, there is a large metric ball $B_{i}$ in $\mathbf{H}^{3} / G_{i}$ whose geometric limit is disjoint from $C\left(\mathbf{H}^{3} / G_{\infty}\right)$. We can find a boundary component of the convex core $C\left(\mathbf{H}^{3} / G_{i}\right)$ and a pleated surface homotopic to it such that a homotopy between them cannot have image disjoint from the ball $B_{i}$. Since the boundary of the convex core is a pleated surface, using a technique similar to a realization of a homotopy by pleated surfaces and negatively curved pleated surfaces due to Thurston, we can get a pleated surface which meets $B_{i}$. We shall also show that by putting a basepoint so that it is mapped to a point in $B_{i}$, the pleated surface converges geometrically to a pleated surface of finite area in $\mathbf{H}^{3} / G_{\infty}$ which is not contained in the convex core, as $i \rightarrow \infty$. This is a contradiction since we can show that all the pleated surfaces of finite area must be contained in the convex core.

Most part of this paper was written during the author's stay at the IHES. The author expresses his hearty gratitude to the IHES for its hospitality and mathematical stimuli, and also to the CNRS and the JSPS for their financial support. He also thanks Frédéric Paulin for his comment which made it possible to remove an unnecessary assumption in the original version of the theorem. 


\section{Preliminaries.}

\subsection{Generalities.}

For a hyperbolic 3-manifold $M$, its convex submanifold that is minimal among those being deformation retracts of the entire manifold $M$ is called the convex core of $M$, and will be denoted by $C(M)$ throughout this paper. Let $G$ be a Kleinian group such that $\mathbf{H}^{3} / G=M$. Then, the convex core of $M$ lifts to the convex hull of the limit set $\Lambda_{G}$, i.e., the minimal closed convex set of $\mathbf{H}^{3}$ whose closure contains $\Lambda_{G}$. The convex hull of $\Lambda_{G}$ coincides with the union of all ideal tetrahedra whose vertices at infinity lie on $\Lambda_{G}$. A Kleinian group $G$, and the corresponding hyperbolic 3-manifold $M$ are said to be geometrically finite when the convex core of $\mathbf{H}^{3} / G$ has finite volume.

The set of points of $M$ where the injectivity radii are less than $\epsilon / 2$ is called the $\epsilon$-thin part of $M$, and its complement the $\epsilon$-thick part of $M$. (We also refer to the thin part or the thick part when the constant $\epsilon$ is of no interest.) We denote the complement of the cusp neighbourhoods of $M$ by $M_{0}$ for some $\epsilon$ less than or equal to the Margulis constant, and call it the non-cuspidal part. When we want to specify the constant $\epsilon$, we call the non-cuspidal part the $\epsilon$-non-cuspidal part.

\subsection{Compact core.}

For an open 3-manifold $M$, its compact sub-3-manifold $C$ is said to be a compact core of $M$ if the inclusion is a homotopy equivalence. Scott proved in [30] that any open 3-manifold with finitely generated fundamental group has a compact core. This result was generalized by McCullough [24] to a relative version, which implies that if $(M, S)$ is a pair such that $M$ has finitely generated fundamental group and $S$ is a subsurface of $\partial M$ which is incompressible in $M$, then there is a compact core $C$ such that the inclusion from $(C, S \cap C)$ to $(M, S)$ is a relative homotopy equivalence. In particular, for a finitely generated (torsion-free) Kleinian group $G$, the quotient $\mathbf{H}^{3} / G$ has a compact core. When $G$ has parabolic elements, it is more convenient to consider a relative compact core of $\left(\left(\mathbf{H}^{3} / G\right)_{0}, \partial\left(\mathbf{H}^{3} / G\right)_{0}\right)$. When $G$ is geometrically finite, the non-cuspidal part of the convex core $C\left(\mathbf{H}^{3} / G\right) \cap$ $\left(\mathbf{H}^{3} / G\right)_{0}$, which we shall denote by $C\left(\mathbf{H}^{3} / G\right)_{0}$, is a relative compact core of $\left(\left(\mathbf{H}^{3} / G\right)_{0}, \partial\left(\mathbf{H}^{3} / G\right)_{0}\right)$.

We should note that a relative compact core $C$ of the non-cuspidal part $M_{0}$ of a hyperbolic manifold can be regarded as a pared manifold with 
paring locus $C \cap \partial M_{0}$. See for instance Morgan [25] for the definition of pared manifold.

Definition 2.1. A Kleinian group $G$ or a hyperbolic 3 -manifold $M$ is said to be freely indecomposable relative to the parabolic subgroups when all the components of the frontier of a relative compact core for $\left(\mathbf{H}^{3} / G\right)_{0}$ are incompressible.

Note that the condition above is equivalent to the following: if $G$ is decomposed into a free product $A * B$, both of whose factors are non-trivial, then there is a parabolic element of $G$ none of whose conjugates are contained in the factors $A, B$.

\subsection{Geodesic and measured laminations.}

The notion of a measured lamination, introduced by Thurston and which we shall discuss briefly below, will be frequently used in this paper. Let $S$ be a hyperbolic surface of finite area possibly with geodesic boundary. A geodesic lamination on $S$ is a closed subset of $S$ consisting of disjoint simple geodesics not meeting the boundary of $S$. The geodesics constituting a geodesic lamination are called the leaves. A sub-lamination of a geodesic lamination $\ell$, which does not admit a proper sub-lamination is called a minimal component of $\ell$. A minimal component which is not a closed geodesic is called an exceptional minimal component. Any leaf contained in an exceptional minimal component is dense in the component, and is called an exceptional minimal leaf. A geodesic lamination endowed with a transverse measure on arcs which is invariant by parallel translation along leaves is called a measured lamination. For a measured lamination $\lambda$, the support of $\lambda$ is the set consisting of points $x$ on $S$ such that any arc transverse to $\lambda$ containing $x$ at its interior has non-zero measure. From now on, we consider only measured laminations with full support: those having transverse measures whose supports are the entire laminations.

The definition of a geodesic lamination and a measured lamination depends on a choice of hyperbolic metric on $S$. Still, if we have two hyperbolic metrics $m_{1}, m_{2}$ on $S$, there is a natural correspondence between the geodesic laminations on $\left(S, m_{1}\right)$ and those on $\left(S, m_{2}\right)$, by taking a geodesic lamination on $\left(S, m_{1}\right)$ to an isotopic one on $\left(S, m_{2}\right)$. Similarly, by isotoping also the transverse measures, we can define a natural correspondence between the measured laminations on $\left(S, m_{1}\right)$ and those on $\left(S, m_{2}\right)$. Therefore, we can consider measured laminations on $S$ without specifying a hyperbolic metric 
by identifying corresponding measured laminations as above.

The set of measured laminations on $S$ endowed with the weak topology with respect to the measures on transverse arcs is denoted by $\mathcal{M L}(S)$, and called the measured lamination space of $S$. The measured lamination space $\mathcal{M L}(S)$ is known to be homeomorphic to the Euclidean space of dimension $6 g-6+2 b$, where $g$ denotes the genus of $S$ and $b$ the sum of the numbers of the punctures and the boundary components. It is also known that the set of weighted simple closed geodesics on $S$ is dense in $\mathcal{M L}(S)$. Refer to Fathi-Laudenbach-Poénaru [16]. The space obtained from $\mathcal{M L}(S) \backslash\{\emptyset\}$ by identifying measured laminations whose transverse measures are scalar multiples of each other is called the projective lamination space and denoted by $\mathcal{P} \mathcal{L}(S)$.

Definition 2.2. We say that a measured lamination is maximal when it is not a proper sublamination of another measured lamination, and that it is absolutely maximal when its support is maximal as a geodesic lamination, i.e., not a proper sublamination of another geodesic lamination. The latter condition is equivalent to saying that each complementary region of the lamination is either an ideal triangle or a once-punctured monogon (or a monogon with a hole when we allow the surface to have boundary).

\subsection{Pleated surfaces and ending laminations.}

Definition 2.3. For a complete hyperbolic surface $(S, m)$ and a hyperbolic 3-manifold $M$, a proper continuous map $f: S \rightarrow M$ is said to be a pleated surface when

1. the length metric on $S$ induced from $M$ coincides with the one induced from the metric $m$, and

2. there is a geodesic lamination $\ell$ on $(S, m)$ such that both $f \mid \ell$ and $f \mid(S \backslash \ell)$ are totally geodesic.

A geodesic lamination or a measured lamination on $S$ which a pleated surface $f$ maps totally geodesically is said to be realized by $f$. In particular, the geodesic lamination $\ell$ in the condition (2) of the definition above is realized by $f$. The geodesic lamination that is minimal among those for which the condition (2) is satisfied is called the pleating locus of $f$.

If there are two homotopic pleated surfaces $f, g: S \rightarrow M$ both realizing the same geodesic lamination $\lambda$, then we have $f(\lambda)=g(\lambda)$ : to be more precise, there is a homeomorphism $h$ of $S$ isotopic to the identity such that 
$f \circ h|\lambda=g| \lambda$. (This was first proved by Thurston [32]. An alternative proof can be derived from Bonahon's argument in [3].)

In [3], Bonahon proved the following theorem.

Theorem 2.4 (Bonahon). Let $G$ be a Kleinian group which is freely indecomposable relative to the parabolic subgroups. Let $C$ be a relative compact core of $\left(\mathbf{H}^{3} / G\right)_{0}$, and $S$ a component of its frontier. Then, either

1. the end $e$ of $\left(\mathbf{H}^{3} / G\right)_{0}$ facing $S$ is geometrically finite, i.e., there is a neighbourhood of $e$ intersecting no closed geodesics, or

2. there is a sequence of essential simple closed curves $\left\{\gamma_{i}\right\}$ on $S$ such that the closed geodesic $\gamma_{i}^{*}$ freely homotopic to $\gamma_{i}$ in $\mathbf{H}^{3} / G$ tends to the end $e$ as $i \rightarrow \infty$.

In the latter case, we say that the end $e$ is simply degenerate.

As a corollary to this theorem, Bonahon showed, using a technique due to Thurston, that $\left(\mathbf{H}^{3} / G\right)_{0}$ can be compactified to a pared manifold so that $\partial\left(\mathbf{H}^{3} / G\right)_{0}$ correspond to the interior of the paring locus.

We now define ending laminations for simply degenerate ends.

Definition 2.5. In the situation above, for a simply degenerate end $e$, a measured lamination $\lambda$ whose projective class is the limit of $\left\{\left[\gamma_{i}\right]\right\}$ in the projective lamination space $\mathcal{P} \mathcal{L}(S)$ is called an ending lamination of $S$.

The definition above depends on the choice of sequence of simple closed curves $\left\{\gamma_{i}\right\}$. Yet, it was also proved by Thurston and Bonahon that if two measured laminations are ending laminations of the same end, then their supports coincide. Furthermore, an ending lamination is always maximal and connected. These facts were first observed by Thurston [32]. Bonahon's result in [3] on the intersection number of simple closed curves whose geodesic representatives tend to an end provides a proof of the former fact. On the other hand, the latter fact is implied from the compactness of the space of marked pleated surfaces (see Canary-Epstein-Green [8]).

It was proved by Bonahon [3] that the following dichotomy holds for a measured lamination on an incompressible surface in a hyperbolic 3manifold.

Proposition 2.6 (Bonahon). Let $G$ be a Kleinian group which is freely indecomposable relative to the parabolic subgroups, and set $M=\mathbf{H}^{3} / G$. Let $\Sigma$ be an incompressible properly embedded surface in $M$, (i.e., embedded 
in such a way that each puncture of $\Sigma$ corresponds to a cusp of $M)$, and $\lambda$ a measured lamination on $\Sigma$. Then, each component $\ell$ of $\lambda$ satisfies one and only one of the following two conditions.

1. There is a pleated surface homotopic to the inclusion of $\Sigma$ realizing $\ell$.

2. Either $\ell$ is a simple closed curve representing a parabolic class of $G$ or there are a simply degenerate end $e$ of $M_{0}$ facing a frontier component $S$ of $M_{0}$ with an ending lamination $\mu$ and a free homotopy from $S$ to a subsurface of $\Sigma$ taking $\mu$ to $\ell$.

The measured lamination $\lambda$ itself can be realized by a pleated surface homotopic to the inclusion of $S$ if and only if all the components are realizable.

For a surface $\Sigma$ as above, the subset of $\mathcal{M L}(\Sigma)$ consisting of all the measured laminations that can be realized by pleated surfaces homotopic to the inclusion is denoted by $\mathcal{R}(\Sigma)$. Thurston showed in [32] that $\mathcal{R}(\Sigma)$ is an open dense subset of $\mathcal{M L}(\Sigma)$.

\subsection{Algebraic and geometric convergence.}

Let $G$ be a Kleinian group. We consider the set of pairs $\{(\Gamma, \phi)\}$ where $\Gamma$ is a Kleinian group isomorphic to $G$ by an isomorphism $\phi$ which maps parabolic elements to parabolic elements. By identifying two pairs $\left(\Gamma_{1}, \phi_{1}\right)$ and $\left(\Gamma_{2}, \phi_{2}\right)$ when the isomorphism $\phi_{2} \circ \phi_{1}^{-1}$ is realized as a conjugation by an element of $P S L_{2} \mathbf{C}$, we obtain the quotient set denoted by $H(G)$. We denote the class represented by $(\Gamma, \phi)$ again by $(\Gamma, \phi)$, slightly abusing notation. By regarding $H(G)$ as a subset of the representation space of $G$ into $P S L_{2} \mathbf{C}$, modulo conjugacy, we can endow $H(G)$ with a topology. This topology is called the algebraic topology, and when a sequence $\left\{\left(G_{i}, \phi_{i}\right)\right\}$ converges to $(G, \psi)$ in $H(G)$ with respect to the algebraic topology, we say that $\left\{\left(G_{i}, \phi_{i}\right)\right\}$ converges to $(\Gamma, \psi)$ algebraically. Sometimes, we also say that $\left\{G_{i}\right\}$ converges to $\Gamma$ algebraically. Evidently, if $\left\{\left(G_{i}, \phi_{i}\right)\right\}$ converges to $(\Gamma, \psi)$ algebraically, then by taking conjugates of $G_{i}$ and $\phi_{i}$ in $P S L_{2} \mathbf{C}$, we can have representations $\left\{\phi_{i}^{\prime}\right\}$ converging to $\psi$.

Since hyperbolic manifolds are aspherical, an isomorphism $\phi_{i}: G \rightarrow G_{i}$ induces a homotopy equivalence between $\mathbf{H}^{3} / G$ and $\mathbf{H}^{3} / G_{i}$. We denote it by $\Phi_{i}$. Similarly, throughout this paper, for any isomorphism between Kleinian groups, we denote an induced homotopy equivalence by the corresponding symbol in the upper case.

For Kleinian groups, there is another notion of convergence, which is called geometric convergence. 
Definition 2.7. Let $\left\{G_{i}\right\}$ be a sequence of Kleinian groups. A Kleinian group $H$ is said to be the geometric limit of $\left\{G_{i}\right\}$ when

1. every element of $H$ is a limit of elements $\left\{g_{i} \in G_{i}\right\}$, and

2. the limit of any convergent sequence $\left\{g_{i_{j}}\right\}$ for a subsequence $\left\{G_{i_{j}}\right\}$ of $\left\{G_{i}\right\}$ is contained in $H$.

From this definition, it is easy to see that if $\left\{G_{i}\right\}$ converges to $H$ geometrically, then the Hausdorff limit of the limit sets $\Lambda_{G_{i}}$ contains $\Lambda_{H}$. It is known that any sequence of non-elementary Kleinian groups, i.e., non-nilpotent Kleinian groups, has a geometrically convergent subsequence. When $\left\{\left(G_{i}, \phi_{i}\right)\right\}$ converges algebraically to $(\Gamma, \psi)$, if we take conjugates of $G_{i}$ so that $\left\{\phi_{i}\right\}$ converges to $\psi$ as representations, the algebraic limit $\Gamma$ is contained in the geometric limit of any subsequence of $\left\{G_{i}\right\}$. Proofs of these facts can be found in Jørgensen-Marden [21].

Geometric limits of Kleinian groups can be interpreted more geometrically using the notion of geometric convergence of Riemannian manifolds in the sense of Gromov. Let $\left\{\left(M_{i}, x_{i}\right)\right\}$ be a sequence of hyperbolic 3-manifolds with basepoints $x_{i} \in M_{i}$. We say that $\left\{\left(M_{i}, x_{i}\right)\right\}$ converges geometrically to a hyperbolic 3 -manifold with basepoint $\left(M_{\infty}, x_{\infty}\right)$ (in the sense of Gromov) when there is a diffeomorphism $\rho_{i}: B_{r_{i}}\left(M_{i}, x_{i}\right) \rightarrow B_{K_{i} r_{i}}\left(M_{\infty}, x_{\infty}\right)$ between the metric balls with radii $r_{i}$ and $K_{i} r_{i}$ centred at $x_{i}$ and $x_{\infty}$ respectively which is a $\left(K_{i}, r_{i}\right)$-approximate isometry with $K_{i} \rightarrow 1$ and $r_{i} \rightarrow \infty$. Here, $\rho_{i}$ is said to be a $\left(K_{i}, r_{i}\right)$-approximate isometry when $d(x, y) / K_{i} \leq d\left(\rho_{i}(x), \rho_{i}(y)\right) \leq K_{i} d(x, y)$ for any $x, y \in B_{r_{i}}\left(M_{i}, x_{i}\right)$.

Suppose that a sequence of Kleinian groups $\left\{G_{i}\right\}$ converges geometrically to $G_{\infty}$. Fix some point $x \in \mathbf{H}^{3}$ and consider its projections $x_{i} \in \mathbf{H}^{3} / G_{i}$ and $x_{\infty} \in \mathbf{H}^{3} / G_{\infty}$ under the universal covering maps. Then, $\left\{\left(\mathbf{H}^{3} / G_{i}, x_{i}\right)\right\}$ converges to $\left(\mathbf{H}^{3} / G_{\infty}, x_{\infty}\right)$ geometrically in the sense of Gromov. Conversely, if hyperbolic 3-manifolds with basepoints $\left\{\left(M_{i}, x_{i}\right)\right\}$ converge geometrically to $\left(M_{\infty}, x_{\infty}\right)$ in the sense of Gromov, then, we can identify the universal coverings of $M_{i}$ and $M_{\infty}$ with $\mathbf{H}^{3}$ in such a way that

1. there is a point $x \in \mathbf{H}^{3}$ projected to $x_{i}$ and $x_{\infty}$ by the universal covering maps,

2. the hyperbolic 3-manifolds $M_{i}$ are isometric to $\mathbf{H}^{3} / G_{i}$, and $M_{\infty}$ is isometric to $\mathbf{H}^{3} / G_{\infty}$ by these identifications, and

3. the Kleinian groups $G_{i}$ converge geometrically to $G_{\infty}$. 
Thus, the notions of geometric convergence of Kleinian groups and geometric convergence of hyperbolic 3-manifolds in the sense of Gromov are equivalent.

Suppose that we are given a sequence of pleated surfaces $\left\{f_{i}:\left(S, m_{i}\right) \rightarrow\right.$ $M_{i}$ \}, where $M_{i}$ is a hyperbolic 3-manifold, and basepoints $x_{i} \in S$. We say that $\left\{f_{i}\right\}$ with basepoints $x_{i}$ converges to a pleated surface $f_{\infty}:\left(S_{\infty}, m_{\infty}\right) \rightarrow$ $M_{\infty}$ with basepoint $x_{\infty} \in S_{\infty}$ when there are a sequence of positive numbers $r_{i}$ going to infinity and an approximate isometries $\bar{\rho}_{i}: B_{r_{i}}\left(\left(S, m_{i}\right), x_{i}\right) \rightarrow$ $B_{K_{i} r_{i}}\left(\left(S_{\infty}, m_{\infty}\right), x_{\infty}\right), \rho_{i}: B_{r_{i}}\left(M_{i}, f_{i}\left(x_{i}\right)\right) \rightarrow B_{K_{i} r_{i}}\left(M_{\infty}, f_{\infty}\left(x_{\infty}\right)\right)$ such that $\left\{\rho_{i} \circ f_{i} \circ \bar{\rho}_{i}^{-1}\right\}$ converges to $f_{\infty}$ uniformly on any compact subset of $S_{\infty}$. Note that in particular, the target manifold $M_{\infty}$ is a geometric limit of $M_{i}$ with basepoint $f_{i}\left(x_{i}\right)$ and $S_{\infty}$ is a geometric limit of $\left(S, m_{i}\right)$ with basepoint $x_{i}$, hence $S_{\infty}$ is homeomorphic to a subsurface of $S$, whose boundary components are essential simple closed curves on $S$.

Recall that a continuous map $f$ from a surface $S$ to a 3 -manifold $M$ is said to be incompressible when the induced homomorphism $f_{\#}: \pi_{1}(S) \rightarrow \pi_{1}(M)$ is injective. Suppose that for a sequence of incompressible pleated surfaces $\left\{f_{i}: S \rightarrow M_{i}\right\}$, there is a positive constant $\epsilon$ and a point $x_{i}$ on $S$ such that the injectivity radii of $M_{i}$ at $f_{i}\left(x_{i}\right)$ are greater than $\epsilon$. Then, it is known that $\left\{f_{i}\right\}$ with basepoints $x_{i}$ converges geometrically to a pleated surface after taking a subsequence. This property is called the compactness of unmarked pleated surfaces. Refer to Canary-Epstein-Green [8] for the proof.

Suppose that hyperbolic 3 -manifolds $M_{i}$ with basepoints $x_{i}$ converge geometrically to a hyperbolic 3 -manifold $M_{\infty}$ with basepoint $x_{\infty}$. We say that closed subsets $C_{i} \subset M_{i}$ converge geometrically to $C_{\infty} \subset M_{\infty}$ when for approximate isometries $\rho_{i}: B_{r_{i}}\left(M_{i}, x_{i}\right) \rightarrow B_{K_{i} r_{i}}\left(M_{\infty}, x_{\infty}\right)$ associated to the geometric convergence $M_{i} \rightarrow M_{\infty}$, the subsets $\rho_{i}\left(C_{i} \cap B_{r_{i}}\left(M_{i}, x_{i}\right)\right)$ converge to $C_{\infty}$ with respect to the Hausdorff topology. A sequence of closed subsets $\left\{F_{i}\right\}$ of a metric space $X$ is said to converge to a closed set $F$ with respect to the Hausdorff topology if and only if there exists a sequence of positive numbers $\epsilon_{i}$ going to 0 such that $F$ is contained in the $\epsilon_{i}$-neighbourhood of $F_{i}$ and $F_{i}$ is contained in that of $F$.

If the distance of $C_{i}$ from the basepoint $x_{i}$ is bounded above, then a geometric limit of $\left\{C_{i}\right\}$ in $M_{\infty}$ always exists after taking a subsequence.

\section{Homotopy between pleated surfaces.}

We shall first review and slightly generalize Thurston's technique of constructing a homotopy between two pleated surfaces, which consists of pleated surfaces and negatively curved pleated surfaces whose definition will be given 
below.

Definition 3.1. Let $M$ be a complete hyperbolic 3-manifold, $S$ a surface of hyperbolic type. An incompressible map $f: S \rightarrow M$ is said to be a negatively curved pleated surface if the following conditions are satisfied.

1. The surface $S$ has a metric with curvature $\leq-1$ whose length function coincides with the one induced from $M$ by $f$.

2. There is a geodesic lamination $\lambda$ on $S$ each of whose complementary regions is either an ideal triangle, or an ideal quadrilateral, or a oncepunctured monogon, or a once-punctured bigon, and $f \mid \lambda$ is totally geodesic.

Similarly, to the case of pleated surface, we say that a geodesic lamination $\mu$ is realized by a negatively curved pleated surface $f$ when $f \mid \mu$ is totally geodesic.

The following appeared in Section 9 of Thurston [32], in the case when both measured laminations $\lambda_{0}$ and $\lambda_{1}$ are realizable by pleated surfaces homotopic to $f$. Its generalization and a fairly detailed explanation can be found in Section 4 of Ohshika [28]. Also, an alternative way to construct a similar homotopy using simplicial hyperbolic surfaces can be found in Canary [7].

Proposition 3.2. Let $M=\mathbf{H}^{3} / G$ be a hyperbolic 3-manifold, $f: S \rightarrow M$ an incompressible embedding of a hyperbolic surface of finite area $S$ into $M$ taking punctures to cusps, and $\lambda_{0}, \lambda_{1}$ measured laminations on $S$. Then, there exist an arc $\alpha$ connecting $\lambda_{0}, \lambda_{1}$ in $\mathcal{M L}(S)$ whose interior is contained in $\mathcal{R}(S)$, the subset of measured laminations realizable by pleated surfaces homotopic to $f$, and a continuous map $H: S \times(0,1) \rightarrow M$ as follows.

1. For all but countably many $s \in(0,1)$, the measured lamination $\alpha(s)$ is absolutely maximal.

2. For any $s \in(0,1)$, if the measured lamination $\alpha(s)$ is not absolutely maximal, then its complement has only one component that is neither an ideal triangle nor a once-punctured monogon. The component is either an ideal quadrilateral or a once-punctured bigon.

3. There is a monotone non-decreasing continuous surjection $\sigma:[0,1] \rightarrow$ $[0,1]$, and for every $t \in(0,1)$, the surface $H(, t)$ is either a pleated surface or a negatively curved pleated surface realizing the measured lamination $\alpha(\sigma(t))$. 
4. For each $s \in[0,1]$, if we set $t_{0}=\inf \sigma^{-1}(s)$ and $t_{1}=\sup \sigma^{-1}(s)$, the surfaces $H\left(, t_{0}\right)$ and $H\left(, t_{1}\right)$ are pleated surfaces provided that they are defined, i.e., $t_{0} \in(0,1)$ or $t_{1} \in(0,1)$.

5. If $\lambda_{j}$ is contained in $\mathcal{R}(S)$ for $j=0$ or 1 , then $H(, t)$ converges to a pleated surface realizing $\lambda_{j}$ as $t \rightarrow j$ uniformly on any compact subset in $S$.

Proof. We shall show how to deal with the case when both $\lambda_{0}$ and $\lambda_{1}$ are not realizable by a pleated surface homotopic to $f$. A measured lamination on $S$ which is not realizable by a pleated surface homotopic to $f$ contains a component which either is an ending lamination or represents a parabolic class, as was remarked in Section 2. Let $C$ be a relative compact core of $\left(\mathbf{H}^{3} / G\right)_{0}$, and $\Sigma$ a component of its frontier. We should note that there are only finitely many isotopy classes of subsurfaces on $S$ whose images by $f$ are freely homotopic to $\Sigma$. This can be proved by the Jaco-ShalenJohannson theory ([19], [20]) as follows. Cut $C$ along $f(S)$ and let $C^{\prime}$ be the resulting possibly disconnected irreducible manifold. If a surface $F$ on $S$ is freely homotopic to $\Sigma$ in $C$, then there is a sequence of essential homotopies $J_{i}(i=0, \ldots, n)$ in $C^{\prime}$ between $F_{i}$ and $F_{i+1}$ such that $F_{0}=F, F_{1}, \ldots, F_{n-1}$ are subsurfaces of $f(S)$ and $F_{n}=\Sigma$. By the Jaco-Shalen-Johannson theory, every homotopy $J_{i}$ can be deformed into a characteristic pair $\Phi$ of $C^{\prime}$, and $F_{0}, \ldots F_{n}$ can be assumed to be pairwise disjoint. Since $\Sigma$ is not an annulus, this characteristic pair $\Phi$ must be an $I$-pair, and there are only finitely many isotopy classes that $F_{0}, \ldots, F_{n-1}$ can represent. Thus, we have shown that there are only finitely many isotopy classes of subsurfaces on $S$ whose images are freely homotopic to $\Sigma$.

Also, by applying the finiteness of maximal parabolic subgroups to the covering of $M$ associated to $f_{\#}\left(\pi_{1}(S)\right)$, we can see that there are only finitely many homotopy classes of simple closed curves on $f(S)$ representing parabolic classes of $G$. Recall that for each end of $M_{0}$, the support of ending lamination is unique. Therefore, the ending laminations of the end $e$ facing $\Sigma$ form a piecewise linear subspace of $\mathcal{M L}(\Sigma)$. (Recall that the measured lamination space has a piecewise linear structure induced from the weight spaces of train tracks. See Penner-Harer [29].) Furthermore, by computing the dimension of the space of measured laminations, we see that for a given measured lamination $\lambda$ on $S$, the set of measured laminations on $S$ whose supports contain the support of $\lambda$ forms a piecewise linear subspace of $\mathcal{M L}(S)$ of positive codimension. 
Therefore, on $S$, the measured laminations that are not realizable by pleated surfaces homotopic to $f$ constitute a subset of $\mathcal{M L}(S)$ consisting of finitely many piecewise linear subspaces of positive codimension. This implies in particular, for any non-realizable measured lamination and a realizable lamination, there is an arc $\alpha$ connecting them whose interior is contained in the set of realizable laminations, $\mathcal{R}(S)$. Furthermore, as was observed by Thurston in [32], there is a union of countably many piecewise linear subspaces of codimension at least one containing all the measured laminations whose complements have a component which is neither an ideal triangle nor a once-punctured monogon. Also, it was shown there that there is a union of countably many piecewise linear subspaces of codimension more than one containing all the measured laminations whose complements contain either more than two components which are neither ideal triangles nor once-punctured monogons, or a component which is none of an ideal triangle, a once-punctured monogon, an ideal quadrilateral, or a once-punctured bigon. Therefore, perturbing $\alpha$, we can assume that all but countably many points on the arc represent absolutely maximal measured laminations and the countably many exceptions have only one exceptional complementary region which is either an ideal quadrilateral or a once-punctured bigon. Thus, we can apply to our situation the lemma in the original form where both measured laminations $\lambda_{0}, \lambda_{1}$ were assumed to be realizable, and get our lemma.

The same argument works even when $S$ has totally geodesic boundary which is mapped to closed geodesics in $M$, or more generally, when the boundary of $S$ is an ideal polygon which is mapped geodesically to $M$. The latter case can be applied to the restriction of pleated surface to a complementary region of a realized lamination. This makes it possible to prove the following lemma on the existence of a bounded homotopy.

Lemma 3.3. There is a constant $D$ depending only on the topological type of $S$ with the following property. Let $\lambda$ be a maximal measured lamination on $S$. Let $f, g: S \rightarrow M$ be two homotopic pleated surfaces both realizing $\lambda$; hence, it can be assumed that $f|\lambda=g| \lambda$. Then, there is a homotopy $H: S \times I \rightarrow M$ between $f$ and $g$ fixing $\lambda$, such that for every $x \in S$, the diameter of $H(\{x\} \times I)$ is bounded by $D$.

Proof. By assumption, we have $f|\lambda=g| \lambda$. Let $U$ be a component of the complement of $\lambda$. Since $\lambda$ was assumed to be maximal, $U$ contains no simple 
closed geodesic at its interior. Therefore, $U$ is either an ideal polygon; or an ideal polygon with a totally geodesic hole or a puncture, which is topologically an annulus; or a twice-punctured disc with totally geodesic boundary; or a once-punctured annulus with totally geodesic boundary; or a pair of pants with totally geodesic boundary. We have only to prove that we can construct a homotopy between $f \mid U$ and $g \mid U$ which moves the points within a bounded distance fixing its frontier. Let us define the complexity $c(U)$ of $U$ to be Area $(U) / \pi$. The proof is by induction on $c(U)$. If $c(U)=1$, then $U$ is either an ideal triangle or a once-punctured monogon, and as $f|U=g| U$, we have the trivial homotopy satisfying the condition of our lemma. We suppose that $c(U) \geq 2$.

Let $\nu_{0}$ and $\nu_{1}$ be maximal geodesic laminations on $U$ realized by $f$ and $g$ respectively. Note that such a lamination consists of finitely many leaves each of whose ends either spirals around a frontier component of $U$ or tends to a puncture or an ideal vertex of $U$ since no leaf of such a lamination accumulates in $U$, since $U$ admits no measured laminations by assumption. Let us consider the case when $U$ is simply connected first. Both $\nu_{0}$ and $\nu_{1}$ give an ideal triangulation of $U$, and there are only finitely many ideal triangulations of $U$. For instance, when $U$ is an ideal quadrilateral, there are exactly two ideal triangulations. Suppose that, $U$ is an ideal quadrilateral and that $\nu_{0}$ and $\nu_{1}$ give the two distinct ideal triangulations. As is shown in the proof of Proposition 3.2 by Thurston (pp. 9.47-48), there is a one-parameter family of ideal quadrilaterals with curvature $\leq-1$ giving a homotopy between $f$ and $g$ fixing $\operatorname{Fr} U$ then. In general, if $U$ is simply connected, any two ideal triangulations can be transformed from one to another by finite steps repeating a change of triangulations on an ideal quadrilateral in each step. Therefore, we can construct a homotopy between $f$ and $g$ by negatively curved pleated surfaces fixing $\operatorname{Fr} U$. Since there is an upper bound, depending only on the number of vertices, for the distance from points in negatively curved polygons to the frontier, and the number of vertices is bounded by a constant depending only on the topological type of $S$, the diameter of $H(\{x\} \times I)$ is bounded by a constant depending only on the topological type of $S$.

Consider now the case when $U$ is not simply connected. Recall that $U$ admits no measured laminations and that $U$ is not a once-punctured monogon, as $c(U) \geq 2$. Therefore, $U$ is either a once-punctured ideal polygon; or a twice punctured disc with totally geodesic frontier; or an annulus one or both of whose boundary components are totally geodesic (that is, an ideal polygon with a hole or a once-punctured annulus); or a pair of pants with totally geodesic boundary. In these cases, as in the case when $U$ is simplyconnected, there are only finitely many ideal triangulations for $U$. If $U$ is a 
once-punctured ideal bigon, there are exactly two ideal triangulations, and if $\nu_{0}$ and $\nu_{1}$ give the two distinct triangulations, we can construct a homotopy fixing $\operatorname{Fr} U$ and the cusp by surface with curvature $\leq-1$ as is shown in [32], similarly to the case of ideal quadrilateral. The diameter of the homotopy is bounded by a universal constant as in the case of ideal quadrilateral. In general, the number of triangulations is bounded by a constant depending only on the number of vertices, hence by a constant depending only on the topological type of $S$. We can transform the ideal triangulation given by $\nu_{0}$ to that given by $\nu_{1}$ by repeating finitely many times the changes of triangulations on either ideal triangles or once-punctured bigons. Hence the diameter of $H(\{x\} \times I)$ is bounded by a constant depending only on the topological type of $S$.

By this lemma, we can refine Proposition 3.2.

Corollary 3.4. In Proposition 3.2, the map $H_{i}$ can be assumed to have the following property. For $s \in(0,1)$, set $t_{0}(s)=\inf \sigma^{-1}(s)$ and $t_{1}(s)=$ $\sup \sigma^{-1}(s)$. Then, for every point $x \in S$ and any $s \in(0,1)$, the diameter of $H_{i}\left(\{x\} \times\left[t_{0}(s), t_{1}(s)\right]\right)$ is bounded by $D$ given in Lemma 3.3.

A boundary component of the convex core is a typical example of pleated surface. Its pleating locus, which we call the bending locus, is the geodesic lamination along which the surface is bent. A transverse measure on the bending locus can be defined using the bending angle so that for an arc $a$ intersecting the bending locus transversely, its measure is equal to the infimum of the geodesic curvature in the ambient hyperbolic 3-manifold of arcs homotopic to $a$ allowing the endpoints to move along leaves. Refer to Epstein-Marden [13] for details. We call the bending locus endowed with such a transverse measure the bending lamination. The bending lamination is empty if and only if the component is totally geodesic.

Note that every complementary region of a bending lamination on the boundary of the convex core is totally geodesic. By adding a maximal system of disjoint simple closed geodesics in the complementary region of a bending lamination to the bending lamination, we get a maximal measured lamination.

\section{The proof of the main theorem.}

Let $\left\{G_{i}\right\}$ be a sequence of Kleinian groups as given in Theorem 1.1 and $G_{\infty}$ its geometric limit. To prove the theorem, we have only to show that every subsequence of $\left\{\left(G_{i}, \phi_{i}\right)\right\}$ has a subsequence for which the conclusion, 
the coincidence of the convex core of the geometric limit and the geometric limit of the convex cores, holds. Since every subsequence of $\left\{\left(G_{i}, \phi_{i}\right)\right\}$ satisfies the hypotheses of the theorem, we have only to show that the original sequence $\left\{\left(G_{i}, \phi_{i}\right)\right\}$ has a subsequence for which the conclusion is valid. Therefore, we are allowed to take a subsequence each time it is necessary in the proof.

Recall that by Bonahon's theorem, $\mathbf{H}^{3} / G$ and $\mathbf{H}^{3} / G_{i}$ can be compactified to pared manifolds $(M, P)$ and $\left(M_{i}, P_{i}\right)$ respectively where the paring loci correspond to the cusps of $\mathbf{H}^{3} / G_{i}$. Although $M$ and $M_{i}$ are homotopy equivalent, they may not be homeomorphic. Still by Jaco-ShalenJohannson theory (see Johannson [20], Jaco-Shalen [19], Jaco [18], and Canary-McCullough [11]), the homotopy equivalences which are not homotopic to homeomorphisms are generated by 'flips' along an embedded annulus in characteristic I-pairs and 'reshuffling' of boundary annuli in solid torus components of the characteristic pair of $M$. As was shown in Canary-McCullough [11], there appear only these two types of characteristic pairs as we assumed that $G$ has no double trouble. Since there is only one way, up to homotopy, to flip in each $I$-pair, and there are finitely many ways of reshuffling in a solid torus component, there are only finitely many marked homeomorphism types among $\left\{M_{i}\right\}$. Furthermore, in the group of self-homotopy equivalences of $\mathbf{H}^{3} / G$, the subgroup of classes represented by homeomorphisms has finite index as was shown in [11]. Therefore, by taking a subsequence and replacing $G$ with a geometrically finite group whose corresponding hyperbolic 3-manifold is homeomorphic to some $\mathbf{H}^{3} / G_{i}$, we can assume that there is a homeomorphism $\Phi_{i}: \mathbf{H}^{3} / G \rightarrow \mathbf{H}^{3} / G_{i}$ induced by $\phi_{i}$.

As was explained in Section 2, there is an approximate isometry $\rho_{i}: B_{r_{i}}\left(\mathbf{H}^{3} / G_{i}, x_{i}\right) \rightarrow B_{K_{i} r_{i}}\left(\mathbf{H}^{3} / G_{\infty}, x_{\infty}\right)$ with $r_{i} \rightarrow \infty$, where $x_{i}$ and $x_{\infty}$ are basepoints obtained by projecting a fixed basepoint $x$ in $\mathbf{H}^{3}$ by the universal covering maps. It is allowed to move $x_{i}$ within distance bounded independently of $i$. In particular, we can assume that $x_{i}$ is contained in $C\left(\mathbf{H}^{3} / G_{i}\right)$ and $x_{\infty}$ is contained in $C\left(\mathbf{H}^{3} / G_{\infty}\right)$.

We fix for the moment a positive constant $\epsilon$ such that $2 \epsilon$ is less than the Margulis constant. We can assume that $\rho_{i}$ maps the $\epsilon$-thin part of $B_{r_{i}}\left(\mathbf{H}^{3} / G_{i}, x_{i}\right)$ into the $2 \epsilon$-thin part of $\mathbf{H}^{3} / G_{\infty}$, and the $2 \epsilon$-thick part into the $\epsilon$-thick part. Whenever we need to take a smaller $\epsilon$ later in the proof, we take a subsequence of $\left\{G_{i}\right\}$ so that this condition here is preserved.

Before starting the proof, we state the following fact which will be used essentially in the proof several times. 
Lemma 4.1. Let $M$ be a complete hyperbolic 3-manifold. Then, the image of every pleated surface of finite area in $M$ is contained in the convex core $C(M)$.

Proof. Let $h: S \rightarrow M$ be a pleated surface of finite area. Consider a maximal geodesic lamination $\lambda$ realized by $h$. Since $\lambda$ is a geodesic lamination on a hyperbolic surface of finite area, each leaf of $\lambda$ is either a closed geodesic or an exceptional minimal leaf or an isolated leaf each of whose ends goes to a cusp or spirals around a leaf of the former two types. A closed geodesic leaf is mapped to a closed geodesic in $M$, hence is contained in the convex core.

Consider an exceptional minimal leaf $l$ of the geodesic lamination. Then, there is an essential closed curve $\gamma_{k}$ consisting of a geodesic arc $l_{k}$ on $l$ and a short geodesic arc $a_{k}$ transverse to $\lambda$ such that $l_{k}$ comes from the different directions to $a_{k}$ at the endpoints with the following two properties: (i) For any $x \in l$, there exists $k_{0}$ such that if $k \geq k_{0}$, then $x$ is contained in $l_{k}$. (ii) The length of $a_{k}$ goes to 0 as $k \rightarrow \infty$. We can take such closed curves $\gamma_{k}$ so that there is a compact set $K$ of $S$ containing all the $\gamma_{k}$ since $l$ does not tend to a cusp. Now, we consider the image $h\left(\gamma_{k}\right)$, which consists of the geodesic $\operatorname{arc} h\left(l_{k}\right)$ and the arc $h\left(a_{k}\right)$. Since the arcs $a_{k}$ are contained in $K$ and length $\left(a_{k}\right) \rightarrow 0$, we see that length $\left(h\left(a_{k}\right)\right) \rightarrow 0$ as $k \rightarrow 0$. Also, at the two endpoints, the tangent vectors of $h\left(l_{k}\right)$ pointing outward viewed from $h\left(l_{k}\right)$ are nearly parallel with opposite directions. Let $\gamma_{k}^{*}$ be the closed geodesic freely homotopic to $h\left(\gamma_{k}\right)$ in $\mathbf{H}^{3} / G_{\infty}$. Then, the maximal distance of the points on $h\left(\gamma_{k}\right)$ from $\gamma_{k}^{*}$ goes to 0 as $k \rightarrow \infty$, as can be easily seen using the elementary hyperbolic geometry. Since any point $x$ on $l$ is contained in $l_{k}$ for sufficiently large $k$, this implies that the distance from $h(x)$ to $\gamma_{k}^{*}$ goes to 0 as $k \rightarrow \infty$. As $\gamma_{k}^{*}$ is contained in $C(M)$, we see that $h(l)$ is also contained in $C(M)$.

Since each end of other leaves either tends to a cusp or spirals towards a compact leaf or an exceptional minimal leaf, all the images of leaves of $\lambda$ are contained in $C(M)$. Finally, since $S \backslash \lambda$ is mapped totally geodesically by $h$, the image of $h$ itself is contained in $C(M)$.

Let $\gamma$ be an element of $G$ such that $\phi_{i}(\gamma)$ converges to a loxodromic element. Since $\left\{G_{i}\right\}$ converges algebraically, conjugating them by uniformly bounded elements of $P S L_{2} \mathbf{C}$, we can assume that all of the $\phi_{i}(\gamma)$ have the same geodesic line of $\mathbf{H}^{3}$ as axes. Take a point $x$ on such a geodesic line. By our choice of $x$, the minimal translation length of $x$ by the elements of $G_{i}$ is bounded by positive constants both above and below as $i \rightarrow \infty$. We 
can assume that the basepoints $x_{i}$ are the projections of this point $x$ by the universal covering maps since the distance between this $x_{i}$ and the original basepoint is bounded above as $i \rightarrow \infty$. Then, the injectivity radius at $x_{i}$ is bounded below by a positive constant as $i \rightarrow \infty$. Consider the geometric limit $C_{\infty}$ of $C\left(\mathbf{H}^{3} / G_{i}\right)$ with basepoint at $x_{i}$. By definition, $C_{\infty}$ is a closed subset of $\mathbf{H}^{3} / G_{\infty}$. Let $\tilde{C}_{\infty}$ be the inverse image of $C_{\infty}$ with respect to the universal covering map $q: \mathbf{H}^{3} \rightarrow \mathbf{H}^{3} / G_{\infty}$. Recall that the limit sets $\Lambda_{G_{i}}$ converge, after taking a subsequence, to a closed set $F$. By the continuity of convex hulls with respect to the Hausdorff topology on the sphere at infinity proved by Bowditch [4], the convex hull of $F$ is equal to $\tilde{C}_{\infty}$, which is the Hausdorff limit (in $\mathbf{H}^{3}$ ) of the convex hulls of $\Lambda_{G_{i}}$. By the definition of geometric convergence, every fixed point of an element in $G_{\infty}$ is a limit as $i \rightarrow \infty$ of fixed points of elements in $G_{i}$. It follows that $\Lambda_{G_{\infty}}$ is contained in $F$. Therefore, the convex hull of $F$, which coincides with $\tilde{C}_{\infty}$, contains the convex hull of $\Lambda_{G_{\infty}}$. By projecting them down to $\mathbf{H}^{3} / G_{\infty}$, it follows that $C_{\infty}$ contains $C\left(\mathbf{H}^{3} / G_{\infty}\right)$. (Refer also to Theorem 7.2 Taylor [31].) What remains to prove is the opposite inclusion: $C_{\infty} \subset C\left(\mathbf{H}^{3} / G_{\infty}\right)$. Suppose, seeking a contradiction, that $C\left(\mathbf{H}^{3} / G_{\infty}\right)$ is a proper subset of $C_{\infty}$.

Consider a component $\Sigma$ of the frontier of $C_{\infty}$. By the definition of geometric convergence, there is a component $\Sigma_{i}$ of the frontier of $C\left(\mathbf{H}^{3} / G_{i}\right)$ and a point $w_{i}$ on $\Sigma_{i}$ such that $\Sigma_{i}$ with basepoint at $w_{i}$ converges to $\Sigma$ geometrically. Recall that every component of the frontier of $C\left(\mathbf{H}^{3} / G_{i}\right)$ is an embedded incompressible pleated surface. By the compactness of unmarked pleated surfaces, the geometric limit $\Sigma$ must also be a pleated surface. The area of $\Sigma_{i}$ is bounded independently of $i$ as the homeomorphism type of $C\left(\mathbf{H}^{3} / G_{i}\right)$ is fixed. This implies that the area of $\Sigma$ is finite.

Therefore, by Lemma 4.1, every component of the frontier of $C_{\infty}$ must be contained in the convex core $C\left(\mathbf{H}^{3} / G_{\infty}\right)$. Thus, from now on until the end of the proof, we assume that there is no component of the frontier of $C_{\infty}$ except those of $C\left(\mathbf{H}^{3} / G_{\infty}\right)$. By our assumption, $C_{\infty} \backslash C\left(\mathbf{H}^{3} / G_{\infty}\right)$ is nonempty. We shall first show that there is no relatively compact component in $C_{\infty} \backslash C\left(\mathbf{H}^{3} / G_{\infty}\right)$.

Suppose, on the contrary, that a relatively compact component $V$ of $C_{\infty} \backslash C\left(\mathbf{H}^{3} / G_{\infty}\right)$ exists. We can assume, by taking a subsequence, that the ball $B_{K_{i} r_{i}}\left(\mathbf{H}^{3} / G_{\infty}, x_{\infty}\right)$ on which $\rho_{i}^{-1}$ is defined, contains $V$ for every $i$. Take any point $y \in V$. Then, since $y$ is contained in $C_{\infty}$, there exists a point $y_{i}$ in $C\left(\mathbf{H}^{3} / G_{i}\right)$ such that $\left\{\rho_{i}\left(y_{i}\right)\right\}$ converges to $y$. There exists an ideal tetrahedron $\tau_{i}$ containing $y_{i}$ whose vertices at infinity lie on $\Lambda_{G_{i}}$. Since $\rho_{i}^{-1}(V)$ is relatively compact and $\tau_{i}$ intersects $\operatorname{Fr} C\left(\mathbf{H}^{3} / G_{i}\right)$ only at its boundary, there exists a geodesic arc $a_{i}$ in $\tau_{i}$ passing through $y_{i}$ whose 
endpoints lie on $\operatorname{Fr} \rho_{i}^{-1}(V) \backslash \operatorname{Fr} C\left(\mathbf{H}^{3} / G_{i}\right)$. Since the diameters of the $\rho_{i}^{-1}(V)$ are bounded above independently of $i$, the lengths of the $a_{i}$ are also bounded above. Therefore, the arcs $\rho_{i}\left(a_{i}\right)$ converge to a geodesic arc $a_{\infty}$ containing $y$ with endpoints lying on the closure of $\operatorname{Fr} V \backslash \operatorname{Fr} C_{\infty}$, which is contained in $C\left(\mathbf{H}^{3} / G_{\infty}\right)$. Since $C\left(\mathbf{H}^{3} / G_{\infty}\right)$ is convex, this implies that $y$ is contained in $C\left(\mathbf{H}^{3} / G_{\infty}\right)$, and we get a contradiction.

More generally, suppose that there is a component $V$ of $C_{\infty} \backslash C\left(\mathbf{H}^{3} / G_{\infty}\right)$ such that the diameter of $V$ relative to $\operatorname{Fr} V$ is bounded, i.e., there is an upper bound for the distances from the points of $V$ to $\operatorname{Fr} V$. Then, by an argument similar to the above, for any $y \in V$, as a limit of the images of geodesic arcs by $\rho_{i}$, we get either a geodesic arc with endpoints on the closure of $\operatorname{Fr} V \backslash \operatorname{Fr} C_{\infty}$ as above or a geodesic ray or a geodesic line containing $y$ with the following property. The geodesic ray has its endpoint on the closure of $\operatorname{Fr} V \backslash \operatorname{Fr} C_{\infty}$, and there is an upper bound for the distance from the points on the ray or the line to $C\left(\mathbf{H}^{3} / G_{\infty}\right)$. Since, we proved above that the limit cannot be such a geodesic arc, we may assume that the limit is either a geodesic ray or a geodesic line. Since a geodesic ray whose end stays within a bounded distance from $C\left(\mathbf{H}^{3} / G_{\infty}\right)$ is lifted to a geodesic ray in $\mathbf{H}^{3}$ with its endpoint at infinity contained in $\Lambda_{G_{\infty}}$, such a geodesic ray or a geodesic line must be contained in $C\left(\mathbf{H}^{3} / G_{\infty}\right)$, and this contradicts the fact that $y$ lies on this geodesic.

Therefore, there is no component of $C_{\infty} \backslash C\left(\mathbf{H}^{3} / G_{\infty}\right)$ whose diameter relative to its frontier is bounded, in other words, for each component $E$ of $C_{\infty} \backslash C\left(\mathbf{H}^{3} / G_{\infty}\right)$ and for any large $R$, there is a point $y_{R} \in E$ such that the closed metric ball $B_{R}$ with radius $R$ centred at $y_{R}$ is contained in $C_{\infty} \backslash C\left(\mathbf{H}^{3} / G_{\infty}\right)$.

Recall that there is a homeomorphism $\Phi_{i}: \mathbf{H}^{3} / G \rightarrow \mathbf{H}^{3} / G_{i}$. We can assume that $\Phi_{i}$ takes the non-cuspidal part of $\mathbf{H}^{3} / G$ to that of $\mathbf{H}^{3} / G_{i}$. Let $T$ be a boundary component of $C\left(\mathbf{H}^{3} / G\right)$. Since $G$ is freely indecomposable relative to the parabolic subgroups, each end of $\left(\mathbf{H}^{3} / G_{i}\right)_{0}$ is either geometrically finite or simply degenerate. The surface $\Phi_{i}\left(T \cap\left(\mathbf{H}^{3} / G\right)_{0}\right)$ is isotopic to a surface $F_{i}$ obtained by connecting (possibly none) components $S_{i}^{1}, \ldots, S_{i}^{p}$ of the frontier of $C\left(\mathbf{H}^{3} / G_{i}\right)_{0}$ (as subsets of $\left.\left(\mathbf{H}^{3} / G_{i}\right)_{0}\right)$ and (possibly none) embedded surfaces $\Sigma_{i}^{1}, \ldots, \Sigma_{i}^{q}$ in neighbourhoods of simply degenerate ends along annuli on $\operatorname{Fr}\left(\mathbf{H}^{3} / G_{i}\right)_{0}$. We let $L_{i}$ be a measured lamination on $T$ obtained as the pull-back of a measured lamination on $F_{i}$ which is the union of the maximal measured laminations $\lambda_{i}^{1}, \ldots, \lambda_{i}^{p}$ realized on the frontier components of the convex cores, ending laminations $\lambda_{i}^{p+1}, \ldots, \lambda_{i}^{q}$ for the simply degenerate ends, and simple closed curves representing parabolic classes cor- 
responding to core curves of the connecting annuli. (It is possible that $p=0$ or $q=p$. Also, there is a choice for $\lambda_{i}^{p+1}, \ldots, \lambda_{i}^{q}$ since only their supports are uniquely determined.) We are going to prove that for some boundary component $T$, we can find a pleated surface homotopic to $\Phi_{i} \mid T$ intersecting $\rho_{i}^{-1}\left(B_{R}\right)$ in a homotopy between two surfaces $T_{i}$ and $f_{i}$ both of which will be defined below.

The first step for that is the following lemma. Let $\Psi: \mathbf{H}^{3} / G \rightarrow \mathbf{H}^{3} / \Gamma$ be a homotopy equivalence induced by the isomorphism $\psi: G \rightarrow \Gamma$.

Lemma 4.2. There are disjoint essential simple closed curves $c_{1}, \ldots, c_{m}$ on $T$, which are independent of $i$, such that each component of $T \backslash\left(c_{1} \cup \ldots \cup c_{m}\right)$ is homeomorphic to a thrice-punctured sphere, with the following properties.

1. Each of $c_{1}, \ldots, c_{m}$ is mapped to a closed curve representing a loxodromic class by all the $\Phi_{i}$ and $\Psi$.

2. For each $i$, there is an arc $\alpha_{i}:[0,1] \rightarrow \mathcal{M L}(T)$ connecting the measured lamination $L_{i}$ defined above and $c_{1} \cup \cdots \cup c_{m}$, which is regarded as a measured lamination by putting a unit weight on each curve.

3. As in Proposition 3.2, for any $s \in(0,1)$, the measured lamination $\alpha_{i}(s)$ either is absolutely maximal or has only one exceptional complementary region that is an ideal quadrilateral or a once-punctured bigon.

4. There is a pleated surface $f_{i}$ homotopic to $\Phi_{i} \mid T$ realizing $c_{1} \cup \ldots \cup c_{n}$, and a half-open homotopy $H_{i}: T \times(0,1] \rightarrow \mathbf{H}^{3} / G_{i}$ with $H_{i}(, 1)=f_{i}$ consisting of pleated surfaces and negatively curved pleated surfaces.

5. There is a monotone non-decreasing continuous surjection $\sigma:[0,1] \rightarrow$ $[0,1]$ such that for $t \in(0,1]$, the surface $H_{i}(, t)$ is either a pleated surface or a negatively curved pleated surface realizing the measured lamination $\alpha_{i}(\sigma(t))$.

6. If $L_{i}$ is realizable by a pleated surface homotopic to $\Phi_{i} \mid T$, then $H_{i}(, t)$ converges as $t \rightarrow 0$ uniformly on compact subsets of $T$ to a pleated surface realizing $L_{i}$ on the boundary of the convex core. Furthermore, the set $\{t \in I \mid \sigma(t)=0\}$ consists only of 0 .

7. If $L_{i}$ is not realizable, then $T$ is divided into subsurfaces $P^{1}, \ldots, P^{k}$ whose interiors are disjoint and each component of whose frontier is mapped to a closed curve homotopic to a cusp of $\mathbf{H}^{3} / G_{i}$, such that 
as $t \rightarrow 0$, either $H_{i} \mid \operatorname{Int} P^{j} \times\{t\}$ converges to a boundary component of the convex core uniformly on any compact subset of $\operatorname{Int} P^{j}$, or the image of $H_{i} \mid \operatorname{Int} P^{j} \times\{t\}$ in $\left(\mathbf{H}^{3} / G_{i}\right)_{0}$ tends to an end of $\left(\mathbf{H}^{3} / G_{i}\right)_{0}$, whose ending lamination is represented by one of $\lambda_{i}^{p+1}, \ldots, \lambda_{i}^{q}$.

Proof. Fix a measured lamination $\mu$ on $T$ which can be realized by a pleated surface homotopic to $\Psi \mid T$. Since the set of measured laminations realizable by pleated surfaces homotopic to $\Psi \mid T$ is open and invariant under scalar multiplication, we can choose a neighbourhood $V$ of $[\mu]$ in $\mathcal{P} \mathcal{L}(T)$ so that every measured lamination in $V$ is realized by a pleated surface homotopic to $\Psi \mid T$. Furthermore, we can choose such a neighbourhood $V$ so that a measured lamination whose projective class is contained in $V$ is also realized by a pleated surface homotopic to $\Phi_{i} \mid T$ for sufficiently large $i$. (Refer for instance to Brock [5] or Ohshika [28] for the proofs of these facts which are parts of the continuity of the length function.) By taking a subsequence, we can assume that this is the case for all $i$. Since the set of projective laminations whose supports are unions of simple closed curves dividing $T$ into pairs of pants is a dense subset in $\mathcal{P} \mathcal{L}(T)$, we can take a disjoint union of simple closed curves $c_{1} \cup \cdots \cup c_{m}$ dividing $T$ into pairs of pants whose projective class lies in $V$, so that none of them are mapped to parabolic classes by $\Phi_{i}$ or $\Psi$. Let $\mathcal{R}_{i}(T)$ denote the set of measured laminations realized by pleated surfaces homotopic to $\Phi_{i} \mid T$. By Proposition 3.2, there is an arc $\alpha_{i}$ connecting $c_{1} \cup \ldots \cup c_{m}$ and $L_{i}$ whose interior is contained in $\mathcal{R}_{i}(T)$, and a map $H_{i}: T \times(0,1) \rightarrow \mathbf{H}^{3} / G_{i}$ satisfying the conditions there.

Note that only the last two conditions in the statement matter now because all the others follow from Proposition 3.2, since $H_{i}(, t)$ converges uniformly to a pleated surface realizing $c_{1} \cup \cdots \cup c_{m}$ as $t \rightarrow 1$. We shall first consider the second but last condition. Suppose that $L_{i}$ is realizable by a pleated surface homotopic to $\Phi_{i} \mid T$. Then, each component of $L_{i}$ neither is an ending lamination nor represents a parabolic element. Hence, by definition, $L_{i}$ is connected and a maximal measured lamination realized on a boundary component of $C\left(\mathbf{H}^{3} / G_{i}\right)$. By Proposition 3.2, the surfaces $H_{i}(, t)$ converge to a pleated surface $k_{i}: T \rightarrow \mathbf{H}^{3} / G_{i}$ realizing $L_{i}$. Since the complement of $L_{i}$ in the boundary component of the convex core is totally geodesic, every pleated surface realizing $L_{i}$ must have the same image as that boundary component. Therefore, $k_{i}$ itself is a homeomorphism onto the boundary component of the convex core realizing $L_{i}$. Furthermore, we can assume that $\sigma(t)=0$ only if $t=0$ by deleting superfluous parameters since the image of the surface $H_{i}(, t)$ is constantly the boundary component of the 
convex core realizing $L_{i}$ while $t$ is in $\{t \mid \sigma(t)=0\}$.

Suppose next that $L_{i}$ is not realizable by a pleated surface homotopic to $\Phi_{i} \mid T$. Recall that $L_{i}$ contains (possibly none) measured laminations $\lambda_{i}^{p+1}, \ldots, \lambda_{i}^{q}$ each of which is homotopic to an ending lamination for some end of $\left(\mathbf{H}^{3} / G_{i}\right)_{0}$. Let $P$ be one of the surfaces $P^{1}, \ldots, P^{k}$ on which lies $\lambda_{i}^{\rho}$ for some $\rho=p+1, \ldots, q$, supposing that $q>p$. Let $t_{j} \in(0,1)$ be numbers converging to 0 . We consider the surface $H_{i}\left(, t_{j}\right)$, which we denote by $\kappa_{j}: T \rightarrow \mathbf{H}^{3} / G_{i}$. By composing an auto-homeomorphism isotopic to the identity of $T$, we can assume that each boundary component of $P$ is a closed geodesic with respect to the negatively curved metric on $T$ induced by $\kappa_{j}$.

Using the fact that $\lambda_{i}^{\rho}$ is maximal and connected on $P$, we can see that by the argument in Section 6.2 in Bonahon [3], if we take a sufficiently small $\epsilon>0$, then $\kappa_{j} \mid P$ can intersect the $\epsilon$-cuspidal part of $\mathbf{H}^{3} / G_{i}$ only at collar neighbourhoods of $\partial P$. Suppose that there is a compact subset of $\mathbf{H}^{3} / G_{i}$ which $\kappa_{j}(P)$ intersects for every $j$, after taking a subsequence. Since the injectivity radius of $\kappa_{j}(P)$ outside neighbourhoods of collars of the boundary is bounded below by a positive constant, then as remarked above, the surfaces $\kappa_{j}$ converge to a pleated surface or a negatively curved pleated surface realizing $\lambda_{i}^{\rho}$ as $j \rightarrow \infty$. This contradicts the fact that $\lambda_{i}^{\rho}$ is an ending lamination. Since two distinct ends cannot have homotopic ending laminations, we can see that $\kappa_{j} \mid P$ cannot tend to an end other than the one whose ending lamination is $\lambda_{i}^{\rho}$.

Therefore, the complement of the collar neighbourhoods of $\kappa_{j}(\partial P)$ in $\kappa_{j}(P)$ tends to the end whose ending lamination is represented by $\lambda_{i}^{\rho}$ as $j \rightarrow \infty$. Each component of $\kappa_{j}(\mathrm{Fr} P)$ must be contained in the cuspidal part of $\mathbf{H}^{3} / G_{i}$ for sufficiently large $j$ since we can choose a neighbourhood of the end having a product structure $P \times \mathbf{R}$ with $\operatorname{Fr} P \times \mathbf{R}$ lying on $\partial\left(\mathbf{H}^{3} / G_{i}\right)_{0}$ whereas $\kappa_{j}(P)$ cannot go far enough without having $\kappa_{j}(\mathrm{Fr} P)$ in the cuspidal part. Thus, we have shown that the intersection of $\kappa_{j}(P)$ and the non-cuspidal part tends to the end whose ending lamination is represented by $\lambda_{i}^{\rho}$.

Next, consider the minimal supporting surface $P$ for a measured lamination $\lambda_{i}^{\rho}$ among $\lambda_{i}^{1}, \ldots, \lambda_{i}^{p}$, supposing $p \neq 0$. As before, let $\kappa_{j}$ be $H_{i}^{\prime}\left(, t_{j}\right)$ and consider the restriction $\kappa_{j} \mid P$ after an isotopy of $T$ making each boundary component of $P$ a closed geodesic with respect to the negatively curved metric on $T$ induced by $\kappa_{j}$. Since $\lambda_{i}^{\rho}$ is maximal on $P$ and each compact leaf of $\lambda_{i}^{\rho}$ represents a loxodromic class of $G_{i}$, as before, by Section 6.2 in Bonahon [3], we can assume that $\kappa_{j}(P)$ intersects the cuspidal part of $\mathbf{H}^{3} / G_{i}$ only at neighbourhoods of the boundary components. Since $\lambda_{i}^{\rho}$ is realized on a boundary component of $C\left(\mathbf{H}^{3} / G_{i}\right)$, it cannot represent an ending lamina- 
tion of an end. (This follows from Proposition 2.4.) Therefore, after taking a subsequence, $\kappa_{j} \mid \operatorname{Int} P$ must converge to a pleated surface $\kappa_{\infty}$ (possibly with boundary) realizing $\lambda_{j}^{\rho}$ uniformly on any compact subset of $\operatorname{Int} P$. Since $L_{i}$ contains each boundary component of $P$ as a leaf, $\kappa_{\infty}$ must map each boundary component of $P$ to a cusp. (Otherwise, a boundary component of $P$ must be mapped to a closed geodesic, which is impossible because it represents a parabolic class.)

Now, recall that each complementary region of $\lambda_{j}^{\rho}$ is totally geodesic on the boundary component of $C\left(\mathbf{H}^{3} / G_{i}\right)$. Therefore, the image of the surface $\kappa_{\infty}$, which realizes $\lambda_{j}^{\rho}$, must coincide with the boundary component.

Lemma 4.3. Let $f_{i}:\left(T, m_{i}\right) \rightarrow \mathbf{H}^{3} / G_{i}$ be a pleated surface homotopic to $\Phi_{i} \mid T$ realizing $c_{1} \cup \cdots \cup c_{m}$ in the precedent lemma. If we take a basepoint in the non-cuspidal part of $\left(T, m_{i}\right)$, then $\left\{f_{i}\right\}$ converges geometrically to a pleated surface $f_{\infty}: T \rightarrow \mathbf{H}^{3} / G_{\infty}$ which can be lifted to a pleated surface $\tilde{f}_{\infty}: T \rightarrow \mathbf{H}^{3} / \Gamma$.

Proof. Let $p: \mathbf{H}^{3} / \Gamma \rightarrow \mathbf{H}^{3} / G_{\infty}$ be the covering map associated to the inclusion $\Gamma \subset G_{\infty}$.

Since $\Psi\left(c_{1}\right), \ldots, \Psi\left(c_{m}\right)$ represent loxodromic classes, the length of the closed geodesic $c_{i}^{j}$ homotopic to $\Phi_{i}\left(c_{j}\right)$ is bounded both above and below by positive constants as $i \rightarrow \infty$ for each $j=1, \ldots, m$. Therefore, any pair of pants in the pants decomposition of $T$ obtained by cutting $T$ along $c_{1} \cup \ldots \cup c_{m}$ converges geometrically to a pair of pants with geodesic boundary as $i \rightarrow \infty$. This implies that $\left\{f_{i}\right\}$ converges to a pleated surface $f_{\infty}$ mapping each $c_{j}$ to the closed geodesic $c_{\infty}^{j}$ which is the limit of $c_{i}^{j}$ if we take basepoints outside the cuspidal parts. The closed geodesic $c_{\infty}^{j}$ must coincide with the image of the closed geodesic homotopic to $\Psi\left(c_{j}\right)$ by the covering projection $p$.

Again since $\Psi\left(c_{1}\right), \ldots, \Psi\left(c_{m}\right)$ represent loxodromic classes of $\Gamma$, there is a pleated surface $g: T \rightarrow \mathbf{H}^{3} / \Gamma$ homotopic to $\Psi \mid T$ realizing $c_{1} \cup \ldots \cup c_{m}$. The pleated surfaces $p \circ g$ and $f_{\infty}$ both realize $c_{1} \cup \ldots \cup c_{m}$ as closed geodesics $c_{\infty}^{1} \cup \ldots \cup c_{\infty}^{m}$. By composing an auto-homeomorphism of $T$ isotopic to the identity to $g$, we can assume that $p \circ g\left(c_{j}\right)=c_{\infty}^{j}$. Recall, on the other hand, that we have $f_{\infty}\left(c_{j}\right)=c_{\infty}^{j}$.

Let $F$ be a pair of pants in the pants decomposition of $T$ by $c_{1} \cup \cdots \cup c_{m}$. Consider an essential arc $a$ connecting one of the boundary components $c_{j}$ with another $c_{j^{\prime}}$ of $F$. (It is possible that $j=j^{\prime}$ when $c_{j}$ is nonseparating.) Moving the basepoint to the endpoint of $a$ on $c_{j}$, we consider the loop represented by $a * c_{j^{\prime}} * a^{-1}$ based at that point. By composing 
auto-homeomorphisms of $T$ isotopic to the identity, we can assume that the length of $f_{i}(a)$ is bounded as $i \rightarrow \infty$ since the lengths of the closed geodesics $c_{i}^{j}$ are bounded below by a positive constant. This implies that $f_{i}\left(a * c_{j^{\prime}} * a^{-1}\right)$ converges geometrically to a loop homotopic to $p \circ g\left(a * c_{j^{\prime}} * a^{-1}\right)$. We can decompose $T$ into hexagons using the pants decomposition and such arcs as $a$ above. As remarked above, $f_{\infty}$ can be homotoped so that on the boundaries of the hexagons, $f_{\infty}$ and $p \circ g$ coincide. Since $\mathbf{H}^{3} / G_{\infty}$ is irreducible, this implies that $f_{\infty}$ and $p \circ g$ are homotopic. Therefore, $f_{\infty}$ can be lifted to a pleated surface to $\mathbf{H}^{3} / \Gamma$.

The pleated surface $\tilde{f}_{\infty}$ has image contained in the convex core $C\left(\mathbf{H}^{3} / \Gamma\right)$ by Lemma 4.1. Let $p: \mathbf{H}^{3} / \Gamma \rightarrow \mathbf{H}^{3} / G_{\infty}$ be the covering map associated to the inclusion $\Gamma \subset G_{\infty}$ as in the proof above. Then, $f_{\infty}(T)=p \circ \tilde{f}_{\infty}(T)$ is contained in $p\left(C\left(\mathbf{H}^{3} / \Gamma\right)\right) \subset C\left(\mathbf{H}^{3} / G_{\infty}\right)$.

Consider the boundary components $T^{1}, \ldots, T^{\mu}$ of $C\left(\mathbf{H}^{3} / G\right)$. For each of them, we can define $f_{i}, f_{\infty}, \tilde{f}_{\infty}$ as above, all of which we denote by $f_{i}, f_{\infty}, \tilde{f}_{\infty}$. The surfaces $T^{1}, \ldots, T^{\mu}$ cobound relative to the cuspidal part the compact submanifold $C\left(\mathbf{H}^{3} / G\right)_{0}$ in $\left(\mathbf{H}^{3} / G\right)_{0}$ representing the fundamental class of $\left(C\left(\mathbf{H}^{3} / G\right)_{0}, \operatorname{Fr}\left(\mathbf{H}^{3} / G\right)_{0}\right)$. Its image $\Psi\left(C\left(\mathbf{H}^{3} / G\right)_{0}\right)$ can be homotoped to a 3-chain $C^{\prime}$ bounded by $\tilde{f}_{\infty}\left(T^{1}\right) \cup \ldots \cup \tilde{f}_{\infty}\left(T^{\mu}\right)$ in $\left(\mathbf{H}^{3} / \Gamma\right)_{0}$ relative to the cuspidal part, which we can assume to be contained in $C\left(\mathbf{H}^{3} / \Gamma\right)$ because it is a deformation retract of $\mathbf{H}^{3} / \Gamma$ and it contains all the $\tilde{f}_{\infty}\left(T^{1}\right), \ldots, \tilde{f}_{\infty}\left(T^{\mu}\right)$.

Recall that, by assumption, for any large $R$, we can take a metric ball $B_{R}$ with radius $R$ in $E$, a component of the complement of $C_{\infty} \backslash C\left(\mathbf{H}^{3} / G_{\infty}\right)$. We can further assume that the centre $y_{R}$ of $B_{R}$ is in the $2 \epsilon$-thick part of $\mathbf{H}^{3} / G_{\infty}$. Let $B_{i}$ be the inverse image $\rho_{i}^{-1}\left(B_{R / 3}\right)$ of the ball $B_{R / 3}$ with radius $R / 3$ concentric with $B_{R}$. Then, $B_{i}$ is disjoint from $f_{i}\left(T^{1}\right), \ldots, f_{i}\left(T^{\mu}\right)$ for sufficiently large $i$ since $B_{R}$ is disjoint from $f_{\infty}\left(T^{1}\right), \ldots, f_{\infty}\left(T^{\mu}\right)$. We can also assume that $B_{i}$ is contained in $C\left(\mathbf{H}^{3} / G_{i}\right)$.

Lemma 4.4. Let $T$ be any one of $T^{1}, \ldots, T^{\mu}$, and let $H_{i}(T, t)$ be the homotopy given by Lemma 4.2. After taking a subsequence, if necessary, we can assume that there exists $t_{0}>0$ such that if $t<t_{0}$ the ball $B_{i}$ is disjoint from $H_{i}(T, t)$ for every $i$.

Proof. Let us suppose, seeking a contradiction, that for each $i$, there is a sequence $\left\{t_{j}\right\}$ going to 0 , which may depend on $i$, such that $H_{i}\left(T, t_{j}\right)$ intersects $B_{i}$ for every $j$. First consider the case when $L_{i}$ is realizable for all $i$ (after taking a subsequence). Then, $H_{i}\left(, t_{j}\right)$ converges, as $j \rightarrow \infty$, uniformly on any compact set of $T$ to a pleated surface $\zeta_{i}: T \rightarrow \mathbf{H}^{3} / G_{i}$ 
realizing $L_{i}$ and intersecting $B_{i}$. Putting a basepoint $y_{i}$ on $T$ whose image is contained in $B_{i}$, the pleated surface $\zeta_{i}$ with basepoint $y_{i}$ converges to a pleated surface of finite area $\zeta_{\infty}: T^{\prime} \rightarrow \mathbf{H}^{3} / G_{\infty}$ whose image intersects $B_{R / 3}$, where $T^{\prime}$ is a subsurface of $T$. This contradicts Lemma 4.1.

Next, consider the case when $L_{i}$ is not realizable. Then, $T$ is divided into subsurfaces $P^{1}, \ldots, P^{n}$ and each one of the $H_{i} \mid\left(\operatorname{Int} P^{k} \times t_{j}\right)(k=1, \ldots, n)$ either converges to a boundary component of the convex core uniformly on any compact set or has non-cuspidal part tending to an end of $\left(\mathbf{H}^{3} / G_{i}\right)_{0}$ as $j \rightarrow \infty$. Since the boundary of $P^{k}$ is mapped into the cuspidal part by $H_{i}\left(, t_{j}\right)$ for large $j$, we can assume by taking a subsequence that there is a point $y^{j}$ on $\operatorname{Int} P^{k}$ whose image by $H_{i}\left(, t_{j}\right)$ intersects $B_{i}$ such that $\left\{y^{j}\right\}$ converges to a point $y_{i}$ on $\operatorname{Int} P^{k}$ as $j \rightarrow \infty$. This cannot happen when the image of the subsurface $P^{k}$ by $H\left(, t_{j}\right)$ tends to an end as $j \rightarrow \infty$. Therefore, we can assume that $H_{i}\left(, t_{j}\right) \mid \operatorname{Int} P^{k}$ converges to a homeomorphism $h_{i}$ from Int $P^{k}$ onto a boundary component of the convex core $C\left(\mathbf{H}^{3} / G_{i}\right)$ as $j \rightarrow \infty$. The pleated surface $h_{i}$ with the basepoint $y_{i}$ converges as $i \rightarrow \infty$ to a pleated surface whose image intersects $B_{R / 3}$ as in the precedent case, and we get a contradiction.

Thus, we have proved that the image of $H_{i}(, t)$ is disjoint from $B_{i}$ for $t$ near 0 . When $L_{i}$ is realizable, we define $T_{i}$ to be the limit of $H_{i}(T, t)$ as $t \rightarrow 0$, which is an embedding onto the boundary component of the convex core. When $L_{i}$ is not realizable, we can assume that for each subsurface $P^{k}$ such that $H_{i}\left(\operatorname{Int} P^{k}, t\right)$ converges to a boundary component of the convex core, $H_{i}\left(\operatorname{Int} P^{k}, t\right)$ is homotopic to an embedding inside a small regular neighbourhood of the boundary of the convex core for sufficiently small $t$. For the other $P^{k}$ 's, the surface $H_{i}\left(\operatorname{Int} P^{k}, t\right) \cap\left(\mathbf{H}^{3} / G_{i}\right)_{0}$ tends to an end as $t \rightarrow 0$. Note that even in this case, $H_{i}\left(\operatorname{Int} P^{k}, t\right)$ is homotopic to an embedding because its non-cuspidal part is homotopic to a frontier component of a relative compact core.

If both $H_{i}\left(P^{k}, t\right)$ and $H_{i}\left(P^{k^{\prime}}, t\right)$ converge to boundary components of $C\left(\mathbf{H}^{3} / G_{i}\right)$ for $k \neq k^{\prime}$, then the limit boundary components are distinct; hence for sufficiently small $t$, the surfaces $H_{i}\left(P^{k}, t\right) \cap\left(\mathbf{H}^{3} / G_{i}\right)_{0}$ and $H_{i}\left(P^{k^{\prime}}, t\right) \cap C\left(\mathbf{H}^{3} / G_{i}\right)_{0}$ are disjoint. Suppose next that $H_{i}\left(P^{k}, t\right) \cap\left(\mathbf{H}^{3} / G_{i}\right)_{0}$ tends to an end. Then, it is clear that for sufficiently small $t$, the surface $H_{i}\left(\left(P_{k}, t\right) \cap\left(\mathbf{H}^{3} / G_{i}\right)_{0}\right.$ is disjoint from $H_{i}\left(P^{k^{\prime}}, t\right)$ which converges to a boundary component of the convex core. When $H_{i}\left(P^{k^{\prime}}, t\right) \cap\left(\mathbf{H}^{3} / G_{i}\right)_{0}$ also tends to an end for $k^{\prime} \neq k$, the end toward which it tends is different from the one toward which $H_{i}\left(P^{k}, t\right) \cap\left(\mathbf{H}^{3} / G_{\infty}\right)_{0}$ tends. Hence for sufficiently small $t$, the surfaces $H_{i}\left(P^{k}, t\right) \cap\left(\mathbf{H}^{3} / G_{i}\right)_{0}$ and $H_{i}\left(P^{k^{\prime}}, t\right) \cap\left(\mathbf{H}^{3} / G_{i}\right)_{0}$ are disjoint. Thus, 
in any case, $H_{i}\left(P^{k}, t\right) \cap\left(\mathbf{H}^{3} / G_{i}\right)_{0}$ and $H_{i}\left(P^{k^{\prime}}, t\right) \cap\left(\mathbf{H}^{3} / G_{i}\right)_{0}$ are disjoint for sufficiently small $t$.

Lemma 4.5. In any thin neighbourhood of $H_{i}\left(P^{k}, t\right) \cap\left(\mathbf{H}^{3} / G_{i}\right)_{0}$, there is an embedding homotopic to $H_{i}\left(P^{k}, t\right) \cap\left(\mathbf{H}^{3} / G_{i}\right)_{0}$. In particular, there are embeddings $P_{i}^{k}(t)$ homotopic to the $H_{i}\left(P^{k}, t\right) \cap\left(\mathbf{H}^{3} / G_{i}\right)_{0}$ which are pairwise disjoint for distinct $k$ for sufficiently small $t$.

Proof. Freedman-Hass-Scott proved in [17] that in a complete Riemannian 3-manifold with (possibly empty) convex boundary, any incompressible least area surface homotopic to an embedding is an embedding itself. In our present situation, we consider a Riemannian metric as follows. First, consider a metric obtained by multiplying a huge scalar to the metric in the complement of a given thin regular neighbourhood of $H_{i}\left(P^{k}, t\right)$. Then, using a bump function, we construct a Riemannian metric which is equal to such a metric outside the regular neighbourhood of $H_{i}\left(P^{k}, t\right)$ and equal to the original hyperbolic metric in a thinner regular neighbourhood. By applying the theorem of Freedman-Hass-Scott, we get a least area surface homotopic to it, which is an embedding contained in the regular neighbourhood. By our construction of the metric, such a least area surface must be contained in the thin regular neighbourhood. We denote such an embedding homotopic to $H_{i}\left(P^{k}, t\right)$ by $P_{i}^{k}(t)$. In particular, $P_{i}^{k}(t) \cap\left(\mathbf{H}^{3} / G_{i}\right)_{0}$ tends to an end as $t \rightarrow 0$, and two such embeddings are disjoint for distinct $k$.

Recall that as $t \rightarrow 0$ either $H_{i}\left(P^{k}, t\right)$ converges to a boundary component of the convex core or its non-cuspidal part tends to an end. In the case when $H_{i}\left(P^{k}, t\right)$ converges to a boundary component of the convex core, the surface $H_{i}\left(P^{k}, t\right)$ is homotopic to such an embedding (disjoint from the other embeddings homotopic to $\left.H_{i}\left(P^{k^{\prime}}, t\right)\right)$ in a thin neighbourhood of the boundary component. Since $B_{i}$ is contained in the interior of the convex core for sufficiently large $i$, we can assume that such a homotopy has image disjoint from $B_{i}$. Suppose that $H_{i}\left(P^{k}, t\right) \cap\left(\mathbf{H}^{3} / G_{i}\right)_{0}$ tends to an end as $t \rightarrow 0$. By Bonahon's theorem [3], there is a relative compact core $C_{i}$ of $\left(\mathbf{H}^{3} / G_{i}\right)_{0}$ such that $\left(\mathbf{H}^{3} / G_{i}\right)_{0} \backslash C_{i}$ is homeomorphic to $\operatorname{Fr} C_{i} \times(0,1)$. We can assume that $C_{i}$ contains the ball $B_{i}$. For $t$ sufficiently near to 0 , both $H_{i}\left(P^{k}, t\right) \cap\left(\mathbf{H}^{3} / G_{i}\right)_{0}$ and the embedding $P_{i}^{k}(t) \cap\left(\mathbf{H}^{3} / G_{i}\right)_{0}$ are contained in a component $E_{i} \cong P^{k} \times(0,1)$ of $\left(\mathbf{H}^{3} / G_{i}\right)_{0} \backslash C_{i}$. Since both of them are incompressible and homotopic to the frontier component facing $C_{i}$, they are homotopic in $E_{i}$. Thus, in particular, $H_{i}\left(P^{k}, t\right)$ and $P_{i}^{k}(t)$ are homotopic outside $B_{i}$ also in this case. 
Let $T_{i}$ be an embedding homotopic to $\Phi_{i}(T)$ obtained by connecting $P_{i}^{k}(t)$ for a small $t$ as above along annuli on $\partial\left(\mathbf{H}^{3} / G_{i}\right)_{0}$ and extending the remaining boundary into components of the cuspidal part which correspond to the cusps of $\mathbf{H}^{3} / G$. For the boundary components $T^{1}, \ldots, T^{\mu}$ of the convex core $C\left(\mathbf{H}^{3} / G\right)$, we get disjoint embeddings $T_{i}^{1}, \ldots, T_{i}^{\mu}$ in this way. By letting $t$ as above be sufficiently small, we can assume that all the $T_{i}^{1}, \ldots, T_{i}^{\mu}$ are disjoint from $B_{i}$, that they cobound in $\left(\mathbf{H}^{3} / G_{i}\right)_{0}$ a submanifold $K_{i}$ containing $B_{i}$ inside, and that there is a homotopy between $H_{i}\left(T^{j}, t\right)$ and $T_{i}^{j}$ disjoint from $B_{i}$.

Lemma 4.6. If we take the radius $R$ of $B_{R}$ to be sufficiently large, then there exists a boundary component $T$ of $C\left(\mathbf{H}^{3} / G\right)$ as follows. Let $H_{i}(T, t)$, with small $t$, be the pleated surface disjoint from $B_{i}$ obtained above. Then, the surface $H_{i}(T, t) \cap C\left(\mathbf{H}^{3} / G_{i}\right)_{0}$ is not homologous to $f_{i}(T) \cap C\left(\mathbf{H}^{3} / G_{i}\right)_{0}$ given in Lemma 4.3 in $C\left(\mathbf{H}^{3} / G_{i}\right)_{0} \backslash \operatorname{Int} B_{i}$ relative to the cuspidal part.

Proof. As above, consider the boundary components $T^{1}, \ldots, T^{\mu}$ of $C\left(\mathbf{H}^{3} / G\right)$. Recall that we have pleated surfaces $f_{i}: T^{j} \rightarrow \mathbf{H}^{3} / G_{i}$ converging geometrically to $f_{\infty}: T^{j} \rightarrow \mathbf{H}^{3} / G_{\infty}$ in Lemma 4.3 and that the thick part of the geometric limit $f_{\infty}\left(T^{1}\right) \cup \ldots \cup f_{\infty}\left(T^{\mu}\right)$, with a suitable orientation on each component, bound a 3-chain $p\left(C^{\prime}\right) \subset C\left(\mathbf{H}^{3} / G_{\infty}\right)$ relative to the cuspidal part. By pulling back $p\left(C^{\prime}\right)$ by $\rho_{i}^{-1}$ and perturbing it, we get a 3 -chain $C_{i}$ bounded by $f_{i}\left(T^{1}\right) \cup \ldots \cup f_{i}\left(T^{\mu}\right)$ relative to the cuspidal part with a suitable orientation on each component. (Note here that every thin part containing a puncture of $f_{i}\left(T^{j}\right)$ is a cusp by our construction of $f_{i}\left(T^{j}\right)$.)

Consider 3 -chains $Z^{1}, \ldots, Z^{\mu}$ realizing a homotopy between the noncuspidal parts of the embedding $T_{i}^{j}$ and $f_{i}\left(T^{j}\right)(j=1, \ldots, m)$. Then, the sum of $C_{i}$ and $Z^{1}, \ldots, Z^{\mu}$ with adequate orientations represent the fundamental class (relative to the cuspidal part) of the compact submanifold $K_{i}$ containing $B_{i}$. (See the paragraph just before this lemma.) Therefore, each point in $B_{i}$ has non-zero algebraic intersection number with either $C_{i}$ or one of $Z^{1}, \ldots, Z^{\mu}$. If it has non-zero algebraic intersection number with $Z^{j}$, then $T_{i}^{j}$ cannot be homologous to $f_{i}\left(T^{j}\right)$ outside $B_{i}$ by the invariance of the algebraic intersection number for homology representatives. As $H_{i}\left(T^{j}, t\right)$ is homotopic to $T_{i}^{j}$ outside $B_{i}$, this implies that also $H_{i}\left(T^{j}, t\right) \cap C\left(\mathbf{H}^{3} / G_{i}\right)_{0}$ cannot be homologous to $f_{i}\left(T^{j}\right) \cap C\left(\mathbf{H}^{3} / G_{i}\right)_{0}$ outside $B_{i}$. By choosing $T$ to be such $T^{j}$, we get the conclusion.

Therefore, there only remains the case when a point of $B_{i}$ has non-zero algebraic intersection number with $C_{i}$, which implies that a point of $B_{R / 3}$ intersects the projection of $p\left(C^{\prime}\right)$ in $\mathbf{H}^{3} / G_{\infty}$. This contradicts the fact that $p\left(C^{\prime}\right)$ is contained in $C\left(\mathbf{H}^{3} / G_{\infty}\right)$ while $B_{R / 3}$ is disjoint from $C\left(\mathbf{H}^{3} / G_{\infty}\right)$. 


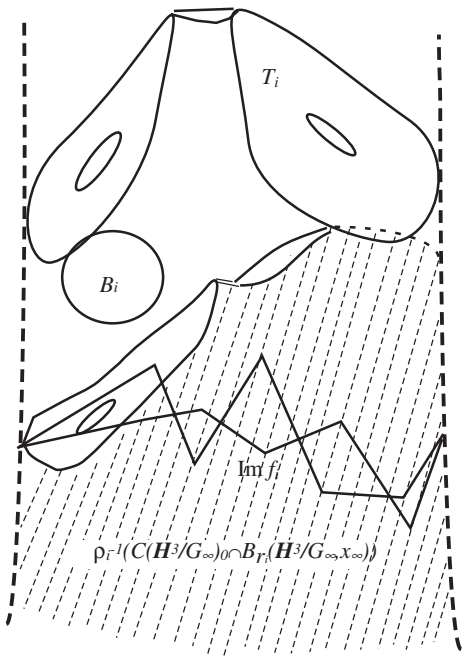

Let $T$ be a boundary component of $C\left(\mathbf{H}^{3} / G\right)$ as given in Lemma 4.6 from now on, and $T_{i}$ an embedding homotopic to $\Phi_{i} \mid T$ defined as before. Denote by $g_{i}$ the surface $H_{i}(, t)$ as above. By taking a subsequence, we can assume that the surface $T$ above does not depend on $i$.

As $g_{i}$ is a surface in the homotopy $H_{i}$ given in Lemma 4.2 , we have a homotopy $H_{i}: T \times[t, 1] \rightarrow \mathbf{H}^{3} / G_{i}$ between $g_{i}$ and $f_{i}$. By Lemma 4.6 and our choice of $T$, the image of the homotopy $H_{i}$ must intersect $B_{i}$. Let $t_{i} \in[t, 1]$ be a point such that $H_{i}\left(T, t_{i}\right)$ intersects $B_{i}$.

Claim 1. If $H_{i}\left(, t_{i}\right)$ realizes a measured lamination $\alpha_{i}\left(\sigma\left(t_{i}\right)\right)$ with $\sigma\left(t_{i}\right) \in$ $(0,1)$, then we are led to a contradiction.

Proof. By Proposition 3.2 and Lemma 4.2, the measured lamination $\alpha_{i}\left(\sigma\left(t_{i}\right)\right)$ either is absolutely maximal or has only one exceptional complementary region that is an ideal quadrilateral or a once-punctured bigon. If $\alpha_{i}\left(\sigma\left(t_{i}\right)\right)$ is absolutely maximal, then $H_{i}\left(, t_{i}\right)$ is an ordinary pleated surface. Otherwise $H_{i}\left(, t_{i}\right)$ may be a negatively curved pleated surface, but still there is $t_{i}^{\prime} \in$ $(0,1)$ with $t_{i}^{\prime}<t_{i}$ such that $H_{i}\left(, t_{i}^{\prime}\right)$ is an ordinary pleated surface realizing the same measured lamination $\alpha_{i}\left(\sigma\left(t_{i}\right)\right)$. In this case, by Corollary 3.4, the diameter of $H_{i}\left(\{x\} \times\left[t_{i}^{\prime}, t_{i}\right]\right)$ is bounded by $D$ for every $x \in T$. We can assume that $D$ is smaller than $R / 3$ choosing a sufficiently large $R$ in the first place. Therefore in either case, we see that there is a pleated surface $h_{i}$ with image intersecting the $R / 3$-neighbourhood of $B_{i}$. Take a basepoint $z_{i}$ on $T$ so that its image by $h_{i}$ is in the $R / 3$-neighbourhood of $B_{i}$. Then $h_{i}$ with 
basepoint $z_{i}$ converges geometrically to a pleated surface $h_{\infty}: T^{\prime} \rightarrow \mathbf{H}^{3} / G_{\infty}$ of finite area with basepoint $z_{\infty}$, where $T^{\prime}$ is a subsurface of $T$. Since $h_{i}\left(z_{i}\right)$ is contained in the $R / 3$-neighbourhood of $B_{i}$, the point $h_{\infty}\left(z_{\infty}\right)$ is contained in $B_{R}$, hence not contained in $C\left(\mathbf{H}^{3} / G_{\infty}\right)$. This contradicts Lemma 4.1.

Claim 2. If $\sigma\left(t_{i}\right)=0$, then we get a contradiction.

Proof. By Lemma 4.2, we have $t_{i}=0$, and $L_{i}$ is realized by $H_{i}(, 0)$ whose image is a boundary component $\Sigma_{i}$ of the convex core $C\left(\mathbf{H}^{3} / G_{i}\right)$. Take a basepoint $y_{i}$ on $\Sigma_{i} \cap B_{i}$. The geometric limit of $\Sigma_{i}$ with basepoint $y_{i}$ becomes a pleated surface intersecting $B_{R}$. This contradicts Lemma 4.1.

Claim 3. If $\sigma\left(t_{i}\right)=1$, then we get a contradiction.

Proof. In this case, for every $x \in T$, its image $f_{i}(x)$ is within distance $D$ from $H_{i}\left(x, t_{i}\right)$ by Lemma 4.2 ; hence $f_{i}(S)$ has a point within distance $D$ from $B_{i}$. Since the non-cuspidal part of $f_{i}(T)$ is contained in $\rho_{i}^{-1}\left(C\left(\mathbf{H}^{3} / G_{\infty}\right) \cap B_{K_{i} r_{i}}\left(\mathbf{H}^{3} / G_{\infty}, x_{\infty}\right)\right)$ for sufficiently large $i$, it must be at a distance more than $R / 3$ from $B_{i}$. Hence, by letting $R$ be larger than $3 D$, we get a contradiction.

Thus, we are led to a contradiction in every case, and have completed the proof of Theorem 1.1.

\section{References.}

[1] J. Anderson and R. Canary, Algebraic limits of Kleinian groups which rearrange the pages of a book, Inventiones Math. 126, (1996), 205-214.

[2] J. Anderson and R. Canary, Cores of hyperbolic 3-manifolds and limits of Kleinian groups II, J. London Math. Soc. (2) 61 (2000), 489-505.

[3] F. Bonahon, Bouts des variétés hyperboliques de dimension 3, Ann. of Math. 124 (1986) 71-158.

[4] B. Bowditch, Some results on the geometry of convex hulls in manifolds of pinched negative curvature, Comment. Math. Helv. 69 (1994), 49-81.

[5] J. Brock, Continuity of Thurston's length function, Geom. Funct. Anal. 10 (2000), 741-797. 
[6] R. D. Canary, On the Laplacian and the geometry of hyperbolic 3manifolds, J. Differential Geom. 36 (1992), 349-367.

[7] R. D. Canary, A covering theorem for hyperbolic 3-manifolds and its applications, Topology 35 (1996), 751-778.

[8] R. D. Canary, D. B. A. Epstein and P. Green Notes on notes of Thurston, Analytical and geometric aspects of hyperbolic spaces London Math. Soc. Lecture Note Ser., Cambridge Univ. Press 111 (1987), $3-92$.

[9] R. Canary and E. Taylor, Kleinian groups with small limit sets, Duke Math. J. 73 (1994), 371-381.

[10] R. Canary and E. Taylor, Hausdorff dimension and limits of Kleinian groups, Geom. Funct. Anal. 9(2) (1999), 283-297.

[11] R. Canary and D. McCullough, Homotopy equivalences of 3-manifolds and deformation theory of Kleinian groups, to appear in Mem. AMS.

[12] T. Comar and E. Taylor, Geometrically convergent Kleinian groups and the lowest eigenvalue of the Laplacian, Indiana Univ. Math. J. 47 (1998), 601-623.

[13] D. Epstein and A. Marden, Convex hulls in hyperbolic space, a theorem of Sullivan, and measured pleated surfaces, Analytical and geometric aspects of hyperbolic spaces, London Math. Soc. Lecture Note Ser. 111 Cambridge Univ. Press (1987), 113-253.

[14] R. Evans, Deformation spaces of hyperbolic 3-manifolds: strong convergence and tameness, Thesis, Univ. Michigan.

[15] C. Fan, Injectivity radius bounds in hyperbolic convex cores I, preprint.

[16] A. Fathi, F. Laudenbach, et V. Poénaru, Travaux de Thurston sur les surfaces, Astérisque, (1979), 66-67.

[17] M. Freedman, J. Hass and P. Scott, Least area incompressible surfaces in 3-manifolds, Invent. Math. 71 (1983), 609-642.

[18] W. Jaco, Lectures on three-manifold topology, CBMS Regional Conference Series in Mathematics, 43, American Mathematical Society, Providence, R.I., (1980). 
[19] W. Jaco and P. Shalen, Seifert fibered spaces in 3-manifolds, Mem. Amer. Math. Soc. 21 (220) (1979).

[20] K. Johannson, Homotopy equivalences of 3-manifolds with boundaries, Lecture Notes in Mathematics, 761 Springer, Berlin, 1979.

[21] T. Jørgensen and A. Marden, Algebraic and geometric convergence of Kleinian groups, Math. Scand. 66 (1990), 47-72.

[22] D. Každan and G. Margulis, A proof of Selberg's hypothesis, Mat. Sb. 75 (117) 1968 163-168.

[23] S. Kerckhoff and W Thurston, Noncontinuity of the action of the modular group at Bers' boundary of Teichmüller space, Invent. Math. 100 (1990), 25-47.

[24] D. McCullough, Compact submanifolds of 3-manifolds with boundary, Quart. J. Math. Oxford Ser. (2) 37 (1986), no. 147, 299-307.

[25] J. Morgan, On Thurston's uniformization theorem for threedimensional manifolds, The Smith conjecture Academic Press (1984), $37-125$.

[26] D. McCullough, A. Miller and G. A. Swarup, Uniqueness of cores of noncompact 3-manifolds. J. London Math. Soc. (2) 32 (1985), 548-556.

[27] C. McMullen, Hausdorff dimension and conformal dynamics I: Strong convergence of Kleinian groups, J. Diff. Geom. 51 (1999), 471-515.

[28] K. Ohshika, Kleinian groups which are limits of geometrically finite groups, to appear in Mem. AMS.

[29] R. Penner and J. Harer, Combinatorics of train tracks, Ann. of Math. Stud., 125, Princeton Univ. Press, Princeton, NJ, 1992.

[30] G. P. Scott, Compact submanifolds of 3-manifolds. J. London Math. Soc. (2) 7 (1973), 246-250.

[31] E. Taylor, Geometric finiteness and the convergence of Kleinian groups, Comm. in Anal. and Geom. 5 (1997) 497-533.

[32] W. Thurston, The geometry and topology of three-manifolds, Princeton Univ., lecture notes. 
Department of Mathematics
Graduate School of Science
Osaka University
Toyonaka, Osaka $560-0043$
Japan
E-mail address: ohshika@math.wani.osaka-u.ac.jp

Received November 22, 2000. 